\title{
TRANSVERSE ISOTACHOPHORESIS USING POLYACRYLAMIDE GEL ELECTRODES
}

\author{
A Thesis \\ presented to \\ the Electrical Engineering Faculty of \\ California Polytechnic State University, San Luis Obispo
}

\author{
In Partial Fulfillment \\ of the Requirements for the Degree \\ Master of Science in Electrical Engineering
}

by

Mark P. Stambaugh

May 2011 
This work performed under the auspices of the U.S. Department of Energy by Lawrence Livermore National Laboratory under Contract DE-AC52-07NA27344.

This document was prepared as an account of work sponsored by an agency of the United States government. Neither the United States government nor Lawrence Livermore National Security, LLC, nor any of their employees makes any warranty, expressed or implied, or assumes any legal liability or responsibility for the accuracy, completeness, or usefulness of any information, apparatus, product, or process disclosed, or represents that its use would not infringe privately owned rights. Reference herein to any specific commercial product, process, or service by trade name, trademark, manufacturer, or otherwise does not necessarily constitute or imply its endorsement, recommendation, or favoring by the United States government or Lawrence Livermore National Security, LLC. The views and opinions of authors expressed herein do not necessarily state or reflect those of the United States government or Lawrence Livermore National Security, LLC, and shall not be used for advertising or product endorsement purposes.

LLNL-TH-471852

(C) 2011

Mark P. Stambaugh

ALL RIGHTS RESERVED 


\section{COMMITTEE MEMBERSHIP}

TITLE: Transverse Isotachophoresis Using Polyacrylamide Gel Electrodes

AUTHOR: Mark P. Stambaugh

DATE SUBMITTED: May 20, 2011

COMMITTEE CHAIR: Vladimir Prodanov

COMMITTEE MEMBER: Bryan Mealy

COMMITTEE MEMBER: Scott Patton 


\begin{abstract}
Transverse Isotachophoresis Using Polyacrylamide Gel Electrodes
\end{abstract}

Mark P. Stambaugh

Separation and isolation of a desired analyte from an impure sample solution containing numerous unwanted interfering agents is the first step of nearly every laboratory test performed in medicine and biology. Nucleic acids are often of particular interest to doctors and researchers, and although methods currently exist for their isolation, these procedures are costly in time, man-power, and real-estate. In addition to easing the execution of presently performed tests, mitigation or elimination of these drawbacks would make a large range of currently unperformed tests both practical and feasible.

This thesis presents a microfluidics-based approach to the isolation of nucleic acids using transverse isotachophoresis (ITP). A boro-silicate glass chip is used with Poly(Acrylamide) gel electrodes to establish an electric field perpendicular to the direction of sample flow, causing a controlled migration of charged particles. The design and fabrication of the microfluidic chip are addressed, along with the development of a transverse-ITP model which predicts the necessary conditions for the successful separation/concentration of an arbitrary sample. Several proof-of-concept images are provided which demonstrate the effectiveness of transverse ITP using surrogate sample inputs.

This thesis proposes a direction for future work which aims toward confirming the model presented and preparing the transverse ITP chip to receive biological samples. 


\section{ACKNOWLEDGEMENTS}

Thanks Maxim, Alex, Bruce, Klint, Jimmy, Julie, and Karon, for all of your help in the lab.

Thanks Patrick, Clinton, Elizabeth, Michael, John, Bishnu, and Thomas. You had nothing to do with my project, but kept me sane throughout. You guys are the best.

Super double-thanks to Ken, who helped me adjust to lab bureaucracy, and also let me use his fume hood to burn off the excess mercury baked to the inside of the lamp after I blew up the bulb (thanks ES\&H for approving). You bailed me out Ken, both times.

Special thanks to Mike and Lisa for letting me crash at their place for the duration of my research. That sure was sweet of you.

I give a nod and a wink to Yvonne, Lani, Nikol, Kirsten, Camille and everyone else behind the EE department desk who handled all of Cal Poly's paperwork and bureaucracy for me from 2006 to 2011. Angels, every last one of you.

Final thanks to Vladimir Prodanov, Bryan Mealy, and Scott Patton for reading through this thesis in its entirety and providing feedback. The slick version you are reading now is the result of their dedication and patience. 
This page, and all the pages which follow, are dedicated to Christine. It is not possible to exaggerate the contribution which she has made to this thesis, despite not working on the project at all. Christine, I would not expect you to understand the indescribably uplifting effect of having a beautiful young woman walk into the lab and make 30 seconds' conversation. The isolation in that room was killing me. You saved my life. Thank you. 


\section{Contents}

LIST OF TABLES.................................................................... ix

LIST OF FIGURES.....................................................................x

LIST OF SYMBOLS....................................................................xii

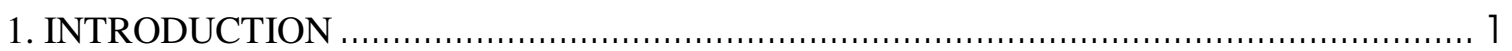

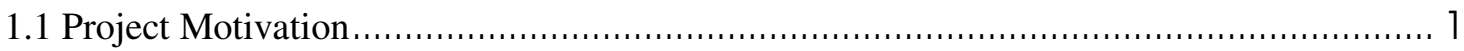

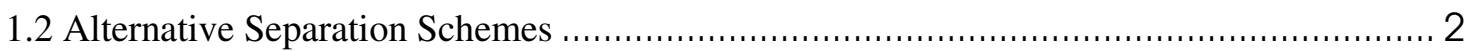

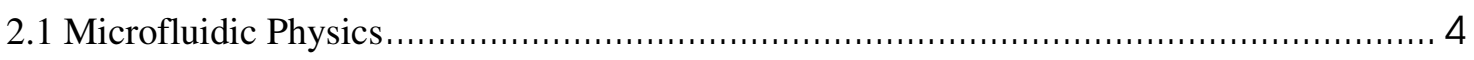

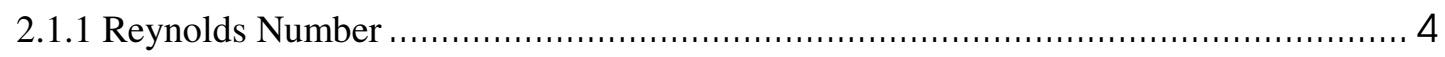

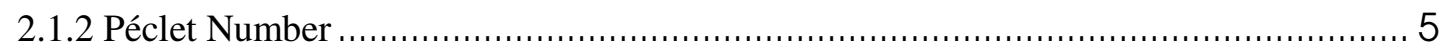

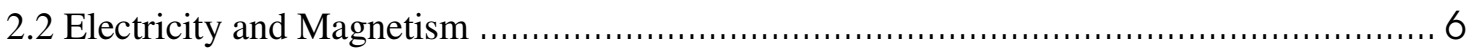

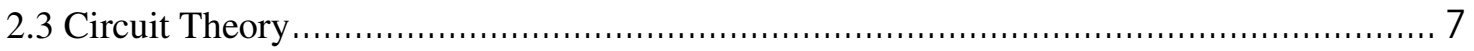

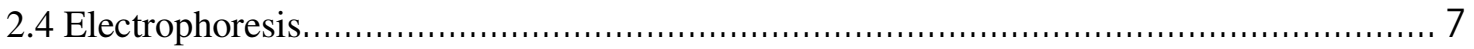

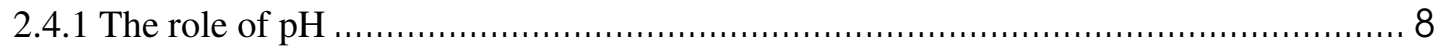

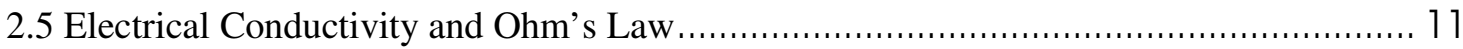

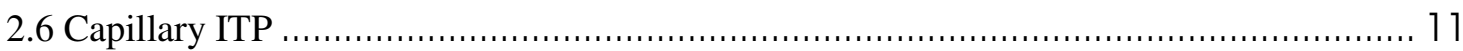

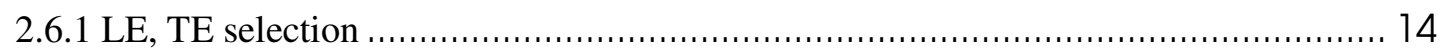

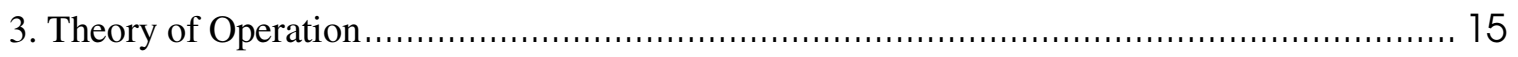

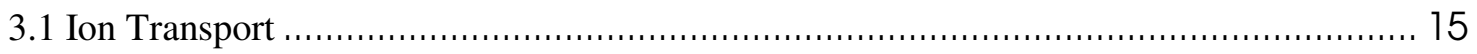

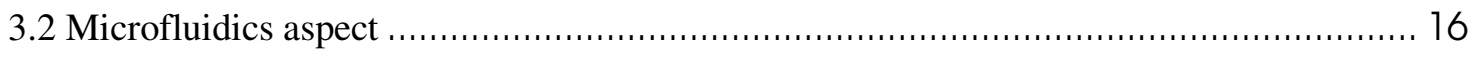

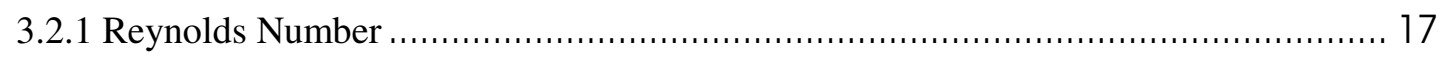

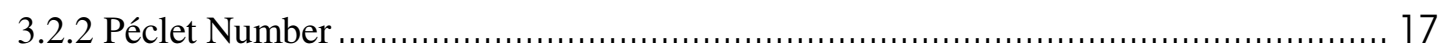

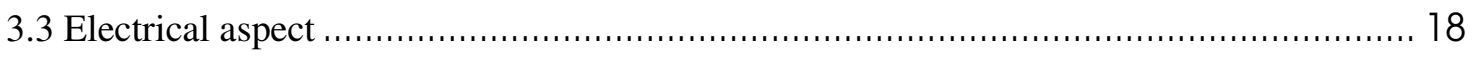

3.3.1 Circuit diagram 
3.3.2 Electric field for low conductivity sample 19

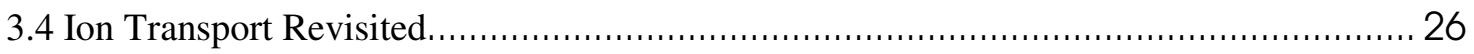

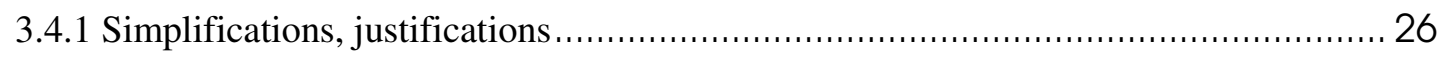

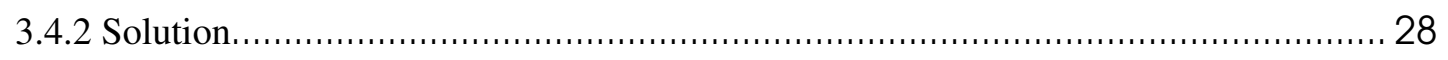

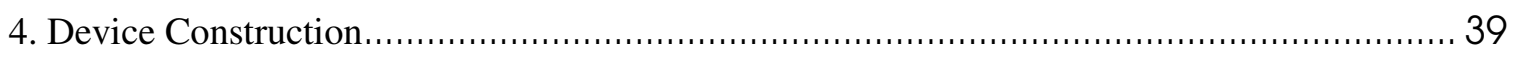

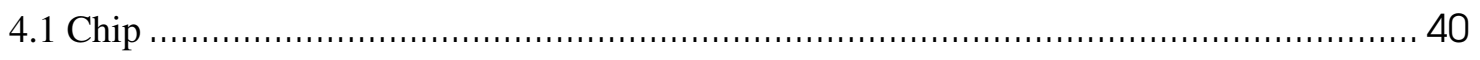

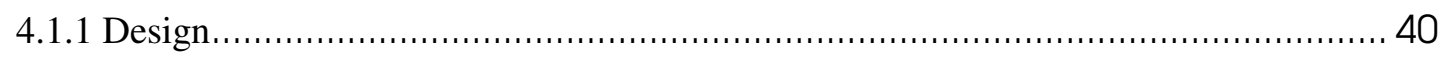

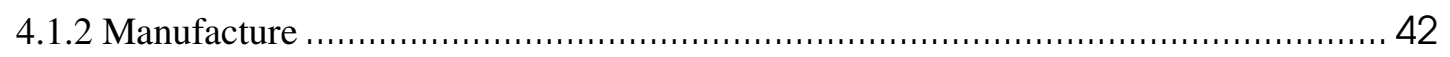

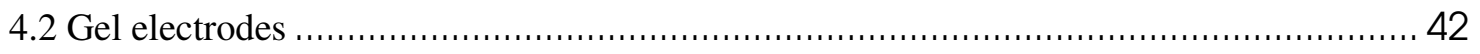

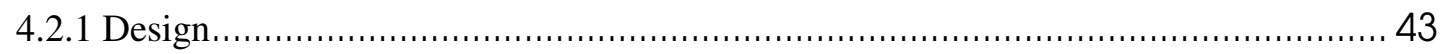

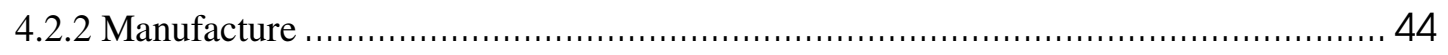

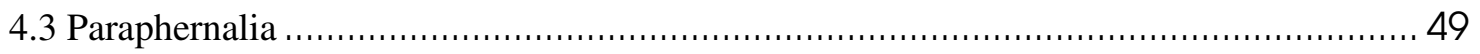

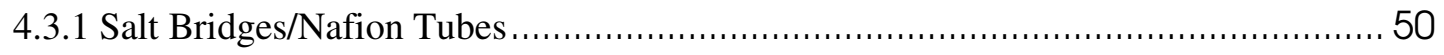

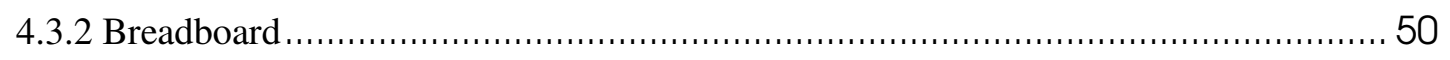

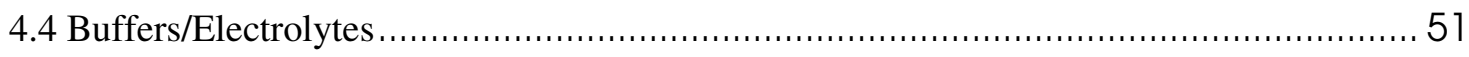

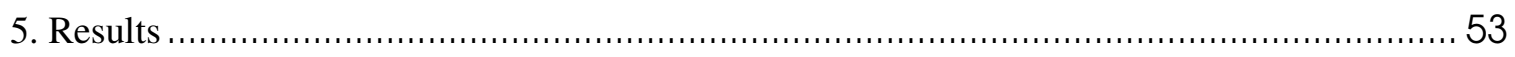

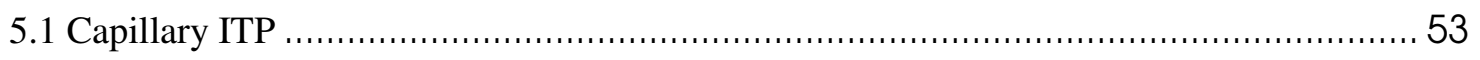

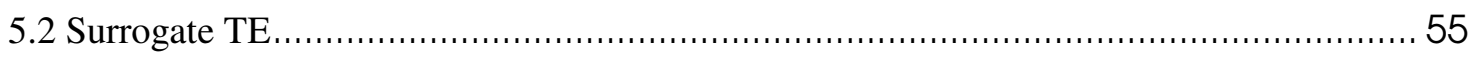

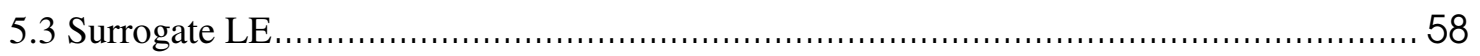

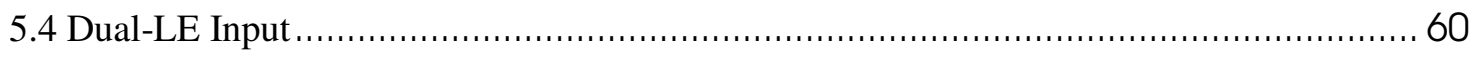

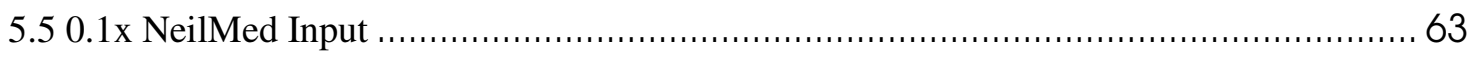

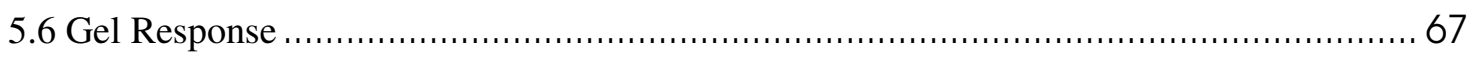

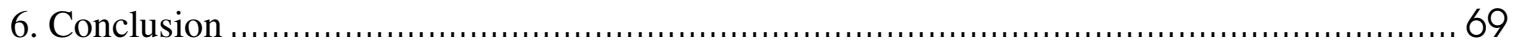

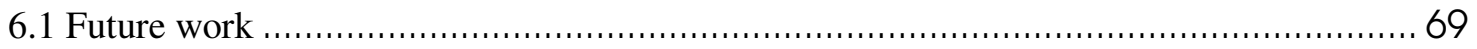

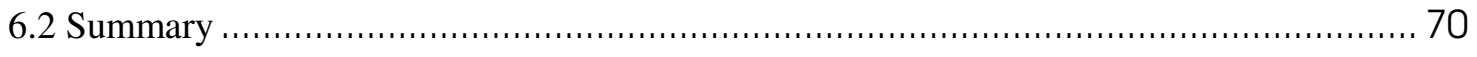




\section{LIST OF TABLES}

Table 1 The effective mobilities and minimum $\mathrm{pH}$ of several species. 14 


\section{LIST OF FIGURES}

Figure 1. The effect of $\mathrm{pH}$ on effective mobility. ........................................... 10

Figure 2. Isotachophoresis in one dimension............................................ 13

Figure 3. The coordinate system to be used in the development of a transverse ITP model.

Figure 4. Circuit-element representation of the ITP system. .............................. 19

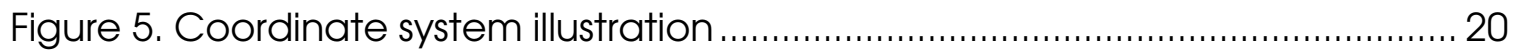

Figure 6. The electric field present in the chip ........................................... 20

Figure 7. Differential element of the high-conductivity (undiluted 1M buffer) LE

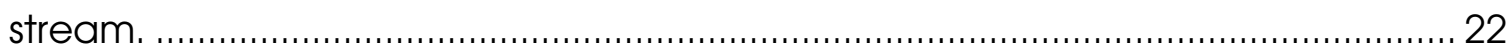

Figure 8. The magnitude of the electric potential along the longest LE channel. 24 Figure 9. The magnitude of the electric potential along the shortest LE channel.25 Figure 10. The coordinate system to be used in the following analysis................. 29

Figure 11. Concentration profile of the Analyte with all equal to $1 . \ldots \ldots \ldots \ldots \ldots . . . . . . .34$

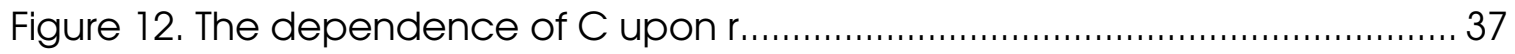

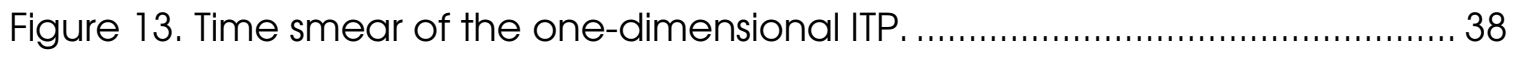

Figure 14. The entire experimental setup.................................................. 39

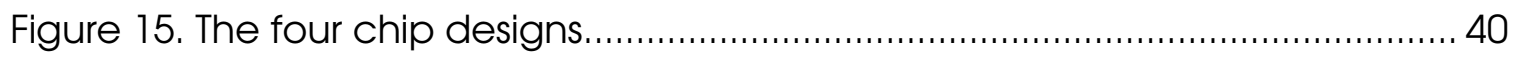

Figure 16. A comparison of chips constructed with, and without posts. ............. 41

Figure 17. Pre- and Post-exposure chips.................................................. 43

Figure 18. A 3-(tri-methoxysylil)propyl methacrylate molecule ........................... 45

Figure 19. An acrylamide molecule....................................................... 45

Figure 20. Emission spectrum of the 350W Mercury short-arc lamp. .................... 47

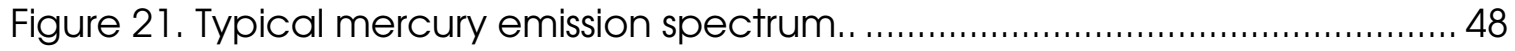

Figure 23. Exposure jig with a chip and mask in place, ready for exposure......... 49

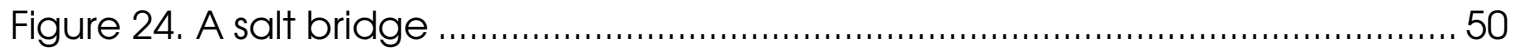


Figure 25. The microfuidics breadboard ............................................................ 51

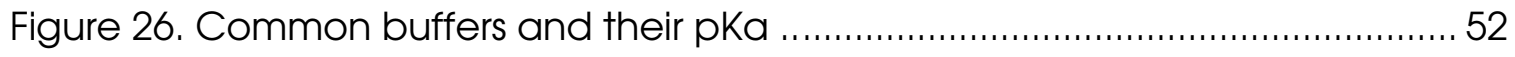

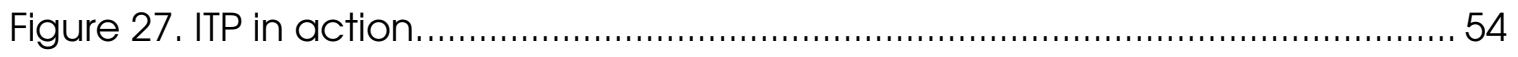

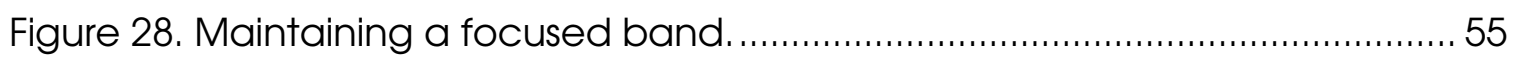

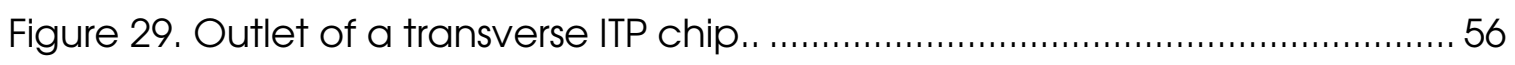

Figure 30. An enlarged view of the 700V frame offigure 29............................. 57

Figure 31. The same device and applied voltage as Figure 29, but run at $75 \%$

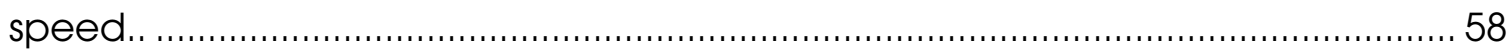

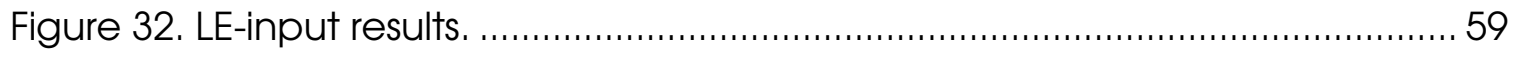

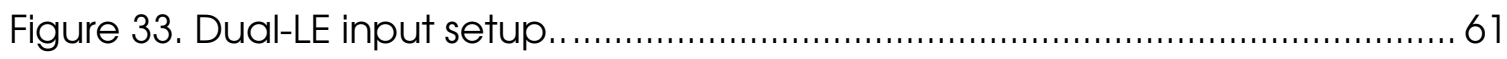

Figure 34. The dual-LE setup under varying voltage ............................................ 62

Figure 35. The expected electrical performance of the 0.1x NeilMed input......... 64

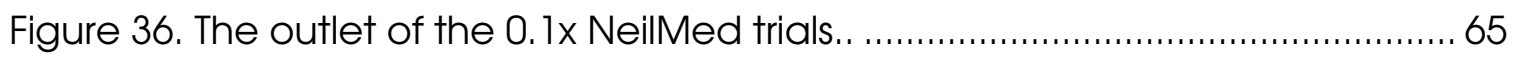

Figure 37. Enlarged view of the 2000V frame of Figure 35 ..................................66 66

Figure 38. Degradation of gel quality at various stages of use ...........................6 67 


\section{LIST OF SYMBOLS}

\begin{tabular}{|c|c|}
\hline $\operatorname{Re}$ & Reynolds Number \\
\hline$\rho$ & Fluid density \\
\hline$\eta$ & Shear viscosity \\
\hline$U_{0}$ & Characteristic velocity \\
\hline$L_{0}$ & Characteristic Length \\
\hline$P e$ & Péclet Number \\
\hline$D_{i}$ & Diffusivity \\
\hline $\bar{\nabla} \bullet$ & Divergence operator \\
\hline$\varepsilon$ & Electrical permittivity \\
\hline $\bar{E}$ & Electric field \\
\hline$\rho_{v}$ & Electric charge density \\
\hline $\bar{\nabla} \times$ & Curl operator \\
\hline $\bar{B}$ & Magnetic Flux density \\
\hline$I$ & Electric current \\
\hline $\bar{J}$ & Electric current density \\
\hline$V$ & Electric Potential \\
\hline$R$ & Electrical Resistance \\
\hline$K_{a}$ & Acid dissociation constant \\
\hline$v$ & Electrophoretic mobility \\
\hline $\bar{v}$ & Effective electrophoretic mobility \\
\hline$\sigma$ & Electrical conductivity \\
\hline$F$ & Faraday's Constant \\
\hline$\rho_{i}$ & Solute concentration \\
\hline$\vec{v}$ & Bulk fluid velocity \\
\hline $\bar{\nabla}$ & Gradient operator \\
\hline$\varphi$ & Electric Potential \\
\hline$l_{T E}$ & Length of the TE region \\
\hline$l_{0}$ & Initial TE length \\
\hline$\gamma$ & Ratio of analyte mobility to TE mobility \\
\hline$t_{s}$ & Necessary time for complete analyte separation \\
\hline$l_{s_{-} \min }$ & Necessary channel length for complete analyte separation \\
\hline$\rho_{0}$ & Maximum analyte concentration \\
\hline$r$ & Ammount of reclaimed sample $(0 \leq r \leq 1)$ \\
\hline$C$ & Analyte concentration factor \\
\hline
\end{tabular}




\section{INTRODUCTION}

\subsection{Project Motivation}

There is a widely held dream in science and medicine, a picture of the ideal way of things. In the dream, a sample containing items of interest is drawn from a host, the sample is analyzed or otherwise experimented upon, and everybody lives happily ever after with the knowledge gained. This is, however, just a dream. In reality, whatever sample is drawn - be it blood, urine, or pond-water - it is impure. The variety of constituents found in blood is extraordinary. Before any useful analysis can be performed, the sample must be filtered of all unwanted interfering agents. We will focus primarily on the example of blood, as it is something which everybody has, and there are almost no afflictions which do not manifest in blood in some way.

Blood is extraordinarily complex. In addition to the usual items of interest: cells, bacteria, viruses, and free-floating DNA, blood contains numerous other agents, such as platelets and proteins, which can only interfere with the analysis of the aforementioned items of interest. Worse still, is that usually only one item from the "interest" list is of particular value at any time, and so the other items all become part of the interfering agents. Thus, before the experimentation and analysis phase can begin, it is crucial to isolate the desired constituent. This process is usually prohibitively expensive as a result of several factors, primarily the need for specialized equipment and reagents, the necessary background of the technician, the controlled environment in which to work, and the time required. Ultracentrifugation of viruses is an example of such a process. It begins with using a regular centrifuge to remove the cells and larger particles from the 
sample, and then finishes with the return of the sample to an ultracentrifuge for a period of several hours. Ultracentrifuges are neither inexpensive nor compact, and the person entrusted to its usage for this purpose is typically not a first-year undergraduate biology student.

This significant expenditure of monetary- and human-resources is the hurdle which the sample preparation project at Lawrence Livermore National Laboratory aims to overcome. By employing a series of three microfluidics-based "virtual filters," the final product has selectively filtered out cells, bacteria, DNA, and viruses, with each constituent being reclaimed and retained as a separate sample. The first stage removes the cells and larger particles, by inducing a standing acoustic wave in the sample stream. The second stage removes the bacteria, by inducing an electric dipole within the bacteria and then forcing migration via electrophoresis. The third stage, which separates the freefloating DNA from viruses, employs transverse isotachophoresis (ITP) to concentrate the DNA and direct it to its own output stream, and it is the development of this stage which this thesis addresses.

\subsection{Alternative Separation Schemes}

There are currently several schemes for sample separation and isolation in common practice, but each is not without its faults. Free-Flow Electrophoresis ${ }^{1}$ separates ionic constituents based on constituent charge, but is highly susceptible to bubble formation via electrolysis. Efforts to impede bubble formation include the introduction of Quinhydrone in solution adjacent to platinum electrodes, but this method 
is not viable past an operating current of $40 \mathrm{uA}$, which severely limits device throughput ${ }^{2}$. Isoelectric Focusing suffers the same electrolysis-induced failure, along with the difficulty of balancing five or more adjacent streams. Photo-polymerized salt-bridges ${ }^{3}$ have been used to isolate the electrodes from the sample region in Isolectric Focusing, but the water-tight seal that the bridges make with the device walls fails if the bridge dries out. 


\section{BACKGROUND}

\subsection{Microfluidic Physics}

The extremely small scales associated with microfluidics serve to separate them entirely from classical fluids in their mathematical and physical treatments. Certain governing forces, derived from both material properties and sample dimensions, which dominate fluid behavior at macroscopic scales, become overshadowed by more subtle forces at the microscopic level. The relative influence of these effects is characterized by a collection of unit-less numbers, the most important of which are the Reynolds (Re) and Péclet $(\mathrm{Pe})$ numbers, which describe the roles of inertial forces, viscous forces, diffusion, and advection. ${ }^{4}$

\subsubsection{Reynolds Number}

The Reynolds number is a ratio of inertial and viscous forces. A high Reynolds number $\left(\operatorname{Re}>3000^{4}\right)$ would describe a fluid dominated by inertial forces, and therefore would be turbulent. A low Reynolds $(\operatorname{Re} \sim \mathrm{O}(10))$ number describes a fluid dominated by viscous forces, manifest as a smooth flow.

The Reynolds Number is defined as

$$
\operatorname{Re}=\frac{\rho U_{0} L_{0}}{\eta}
$$

where $\rho$ is fluid density, $\eta$ is shear viscosity, and $U_{0}$ and $L_{0}$ are characteristic fluid velocities and lengths associated with the flow. These "characteristic values" are derived from measurable parameters, although the definition is not universal across systems, 
making the Reynolds number situationally indicative of fluid behavior. In microfluidics, fluids in the $10<\operatorname{Re}<3000$ region are not conveniently classified or modeled, but that range is dependent on system geometry. For macro-scale pipes the equivalent range would be $2000<\operatorname{Re}<100,000$. Typical values of $U_{0}$ and $L_{0}$ in the microfluid regime are $100 \mu \mathrm{m} / \mathrm{s}$ and $100 \mu \mathrm{m}$, firmly placing most aqueous solutions $(\eta=0.01 \mathrm{~g} / \mathrm{cm} \mathrm{s}, \rho=$ $1 \mathrm{~g} / \mathrm{cm}^{3}$ ) in the $\mathrm{Re}<10$ region. As such, turbulent flow is almost non-existent.

In the case of non-circular channels, Lo is sometimes replaced with the Hydraulic Diameter. For this thesis the difference in predicted operation is unchanged by the minor variations in the Reynolds and Péclet numbers resulting from such a substitution.

\subsubsection{Péclet Number}

The Péclet number is a ratio of the relative timescales for the transport of particles in the fluid via diffusion and convection. A high Péclet number $(\mathrm{Pe}>100) 4$ indicates that a relatively long channel is needed for fluids to mix or homogenize by diffusion alone. A low Péclet number $(\mathrm{Pe}<10)$ dictates a fluid in which diffusion is an effective method for mixing or homogenization over relatively short distances or times.

The Péclet Number is calculated as

$$
P e=\frac{U_{0} L_{0}}{D}
$$

where $D$ is the diffusivity of the solute and $U_{0}$ and $L_{0}$ are characteristic fluid velocities and lengths, as with the Reynolds number. $L_{0}$ is typically the width of the channel, in which case the Péclet number is the necessary length of the channel as a multiple of its 
width (for complete transverse diffusion). Using $U_{0}$ and $L_{0}$ of $100 \mu \mathrm{m} / \mathrm{s}$ and $100 \mu \mathrm{m}$, and a range of typical Diffusion constants from $0.02 \mu \mathrm{m}^{2} / \mathrm{s}$ (cells) to $2000 \mu \mathrm{m}^{2} / \mathrm{s}$ (ions), the Péclet number in the microfluidics regime ranges from 5 to $5 \times 10^{5}$, making diffusion as a mixing method either incredibly effective or wildly impractical, depending solely on the diffusing species.

\subsection{Electricity and Magnetism}

Two of Maxwell's equations are of particular value in the analysis of transverse ITP. They are Gauss's Law of the Electric Field, and Faraday's Law. Gauss's Law of the Electric Field in its differential form is given as

$$
\bar{\nabla} \bullet \varepsilon \bar{E}=\rho_{v}
$$

where $\bar{E}$ is the electric field, $\rho_{v}$ is the space charge, and $\varepsilon$ is the electric permittivity. This equation dictates how the electric field changes in the presence of space charge. The same Law in its integral form is given as

$$
\oint_{S} \varepsilon \bar{E} \bullet d \bar{s}=\int_{V} \rho_{v} d v
$$

where $\mathrm{S}$ is the surface enclosing volume V. In the integral form, this law can be used to determine the charge density at the interface between two regions of different permittivity.

Faraday's Law in its differential form is given as 


$$
\bar{\nabla} \times \bar{E}=-\frac{d \bar{B}}{d t}
$$

where $\bar{B}$ is the magnetic flux density. Faraday's law dictates the effect of a time-varying magnetic field on the electric field.

\subsection{Circuit Theory}

The most useful idea from classical circuit theory is Kirchhoff's Current Law (KCL), given in discrete form as

$$
\sum I=0
$$

and in differential form as

$$
\bar{\nabla} \bullet \bar{J}=0
$$

where $I$ and $\bar{J}$ are current, and current density.

Ohm's law also makes an appearance as

$$
V=I R
$$

Where $V$ is voltage (electrical potential difference), $I$ is electrical current, and $R$ is electrical resistance.

\subsection{Electrophoresis}

Charged objects experience a force in an electric field. Ions dissolved in solution respond to this force by moving within the solution, until reaching such a point where the 
field is zero. The speed with which ions or other charged particles move in solution is given by

$\vec{v}=v \vec{E}$, where $\vec{E}$ is electric field, $\vec{v}$ is velocity, and $v$ is the electrophoretic mobility of the species in question. Electrophoretic mobility is a function of several factors, most notably object size, object charge, and fluid viscosity. Charged particles in solution tend to not be consistently charged over large timescales. For example, a single specimen of a typical weak acid may transition from un-reacted acid (electrically neutral) to conjugate base (negative charge) and back again several $\left(10^{9}\right)$ times per second. For this reason it is convenient to define $\bar{v}$, the time-average electrophoretic mobility. The ergodic theorem states that the time-average value of a specimen should be the same as the ensemble average of multiple identical specimens taken at a single instant, so $\bar{v}$ equals the ensemble average of the electrophoretic mobilities of all such specimens in solution.

\subsubsection{The role of $\mathrm{pH}$}

The $\mathrm{pH}$ value is an indirect factor of electrophoretic mobility, and of particular interest, as it plays an extremely powerful role in determining the specimen's average charge $^{5}$, and is the most convenient solution parameter to alter. In the range $2<\mathrm{pH}<12$, $\mathrm{pH}$ is approximately equal to

$$
p H=-\log _{10} \frac{\left[H_{3} O^{+}\right]}{1 M}
$$


where the brackets denote concentration in Molarity. In all further instances of this and similar calculations, the $1 \mathrm{M}$ is implied but not shown. It is useful now to introduce the number Ka, which varies with species, and is defined as

$$
K_{a}=\frac{\left[A^{-}\right]\left[H_{3} O^{+}\right]}{[A H]}
$$

where $\mathrm{AH}$ is the acid form and $\mathrm{A}^{-}$is the conjugate base of the specimen of interest. Ka describes the equilibrium conditions for the reaction

$$
\mathrm{AH}+\mathrm{H}_{2} \mathrm{O} \Leftrightarrow \mathrm{A}^{-}+\mathrm{H}_{3} \mathrm{O}^{+}
$$

and can take values over several orders of magnitude for different acidic species, and so it is often convenient to define

$$
p K_{a}=-\log K_{a}=\log \frac{[A H]}{\left[A^{-}\right]}+p H
$$

It is plain now how altering the $\mathrm{pH}$ of the solution can dramatically affect the ratio of charged to uncharged specimens, thus enabling incredible control of the average mobility. In the current example, there are only two allowable charge states: 0 and -1 . The electrophoretic mobility of the uncharged particle is zero, and for the negatively charged particle is $v$.

$$
\bar{v}=\frac{[A H]}{[A H]+\left[A^{-}\right]}(0)+\frac{\left[A^{-}\right]}{[A H]+\left[A^{-}\right]} v
$$

Or

$$
\bar{v}=\frac{v}{1+\frac{[A H]}{\left[A^{-}\right]}}=\frac{v}{1+10^{p K_{a}-p H}}
$$


Figure 1 shows that for $p K_{a}-p H \geq 1, \bar{v} \cong 0$, and for $p K_{a}-p H \leq-1, \bar{v} \cong v$.

At $p K_{a}=p H, \bar{v}=\frac{v}{2}$.

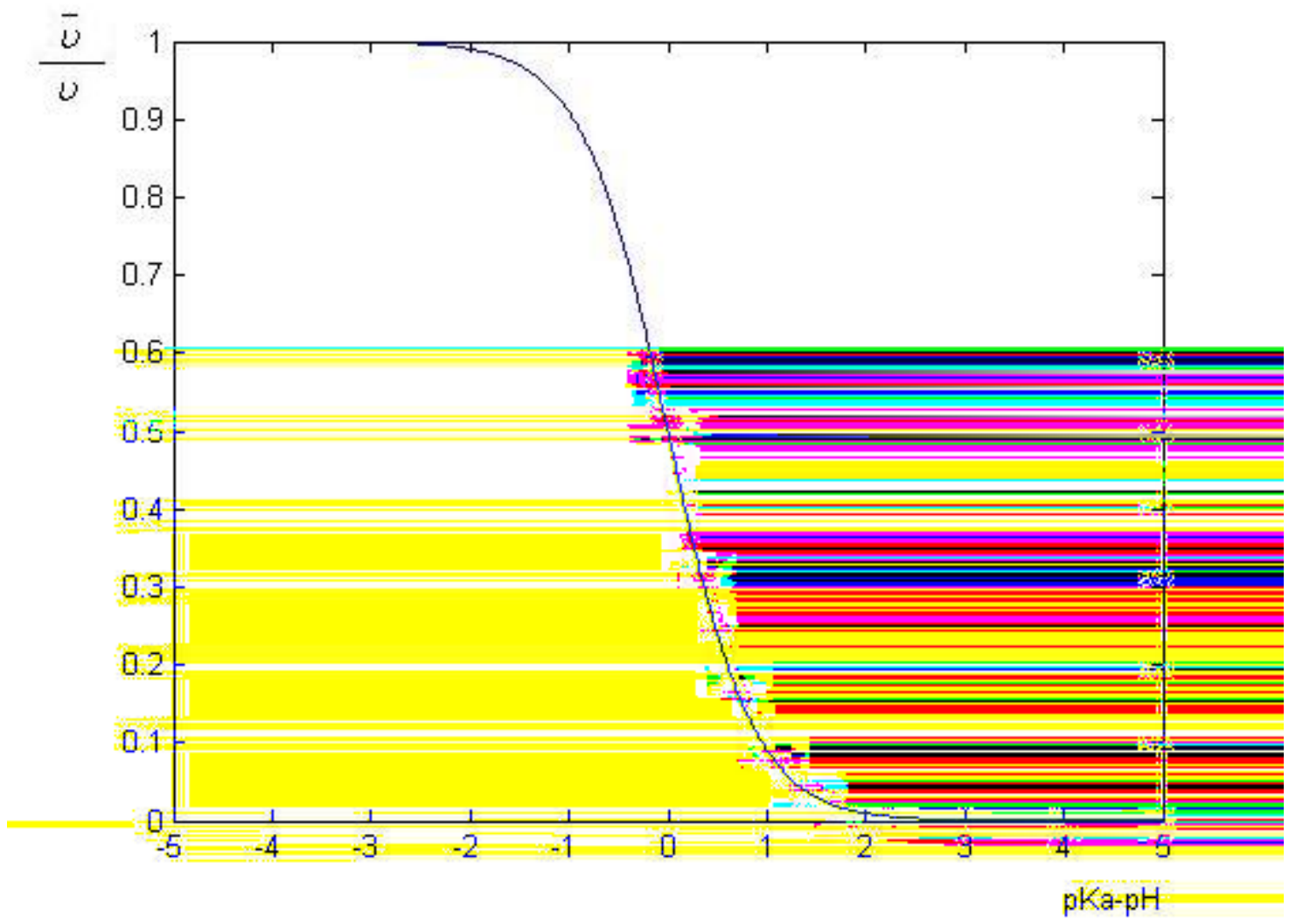

Figure 1. The effect of $\mathrm{pH}$ on effective mobility. At pKa-pH $>1$ and $\mathrm{pKa}-\mathrm{pH}<-1$, the effective mobility can be well approximated as zero or full mobility.

The utility of this effect is apparent when one recalls the low Re condition of microfluidic flow, which enables adjacent streams with differing $\mathrm{pH}$ to remain separate, effectively defining distinct physical regions of higher or lower mobilities. The active 
elements of the buffer solutions can also be tailored to have high Pe, which will prevent unwanted diffusion from interfering with established $\mathrm{pH}$ regions.

\subsection{Electrical Conductivity and Ohm's Law}

Electrical Conductivity is given by

$$
\sigma=F \sum_{i}[i] \overline{v_{i}}
$$

where $F=96,500 \frac{C}{m o l}$ is Faraday's constant, and $i$ takes the values of all charged species within the solution. At any one time there are unthinkably many different ions in solution, but the majority of them are not present in high enough concentration to noticeably contribute to the conductivity.

With conductivity defined in this manner, I introduce Ohm's law in Vector form, given by

$$
\bar{J}=\sigma \bar{E}
$$

\subsection{Capillary ITP}

Traditional Isotachophoresis (ITP) occurs in a microfluidic capillary, effectively a one-dimensional space. There are three species of interest: the Leading Electrolyte (LE), Trailing Electrolyte (TE), and target species, whose electrophoretic mobilities all have the same sign. The LE and TE are chosen to have higher (LE) and lower (TE) 
electrophoretic mobilities than the target species. The LE and TE may even be the same buffer titrated with different titrants to different pHs. The capillary is first flooded with LE from a well at one end, then a well at the other end is filled with a solution of TE and dilute target species. A voltage is applied between the two wells, which establishes an electric field along the length of the adjoining capillary. All three species of interest will move down the capillary due to the presence of the field. The LE always remains ahead of the target species in the capillary, and the TE always follows behind the target species, due to their differing electrophoretic mobilities. This forms an envelope, in which the target species is forced to form a concentrated band as it travels along the capillary, as Figure 2 shows. If ever the TE and target species overlap via diffusion or any other mechanism (as they did in the well prior to voltage application), the higher mobility of the target species immediately corrects the overlap by accelerating the lagging target specimens until they are back in their own band. The same mechanism also keeps the LE out of the target species band. 

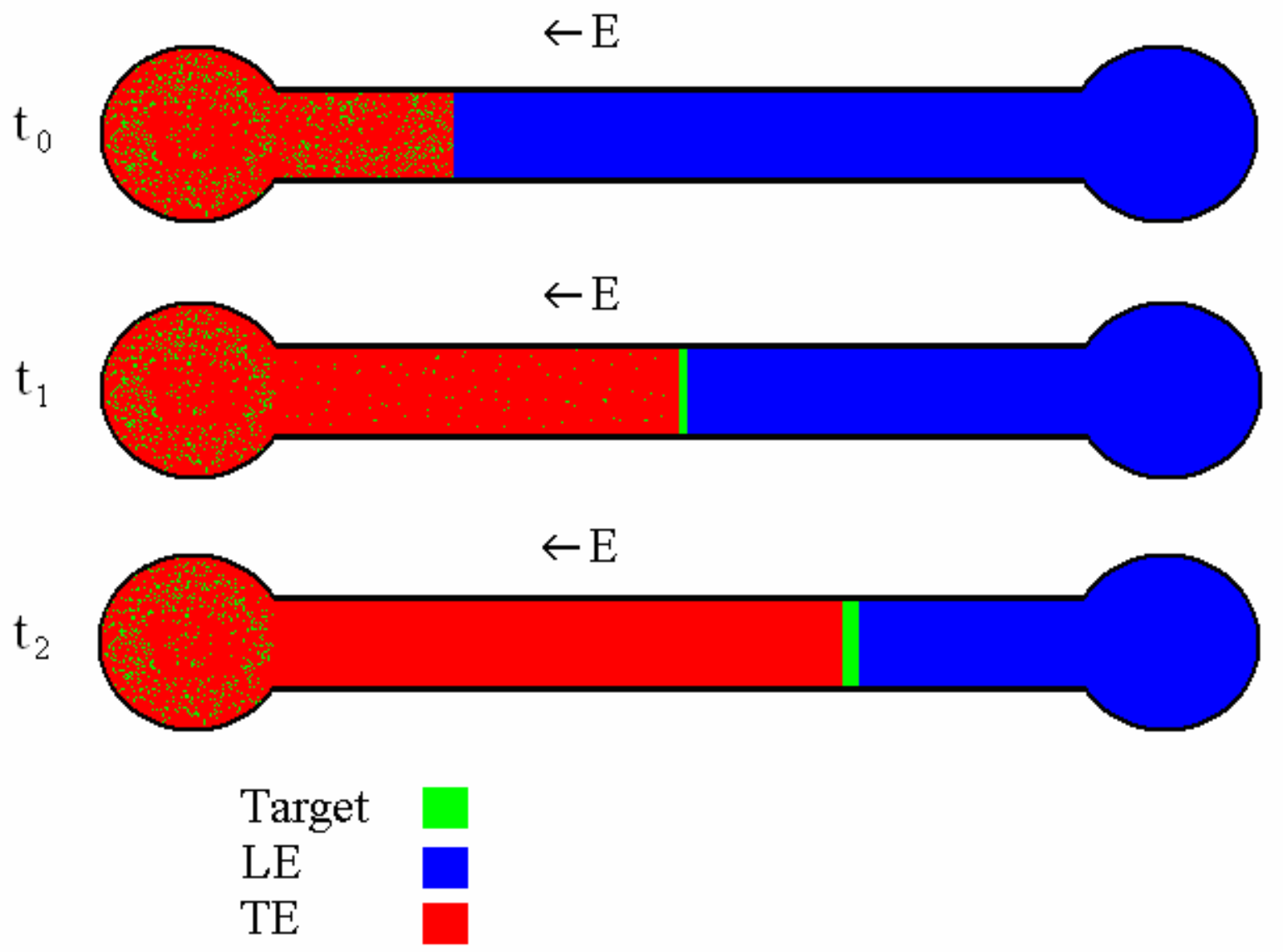

Figure 2. Isotachophoresis in one dimension. There is no electric field in the wells at the ends of the capillary.

$\vec{J}$ is constant throughout the channel (Equation 2.7, 1-D approximation), so at any point in the channel, higher conductivity directly equates to lower field strength (eq. 2.16) and lower electrolyte velocities. This prevents the target species from "bunching up" far ahead of the TE, and the LE from "bunching up" far ahead of the target species. The result is a target species band of consistent width travelling the length of the capillary at near-constant velocity (for a given voltage and LE, TE concentrations). Capillary ITP has been employed in practice with great success ${ }^{6,7}$ as a dilute-sample concentrator, with concentration increases as high as one million ${ }^{8}$. 


\subsubsection{LE, TE selection}

Table 1 contains the electrophoretic mobilities of buffers, titrants, dyes, and organic species relevant to this thesis. Also given are the conditions for which the given mobilities are valid. All experiments performed for this thesis use Fluorescein because it is less expensive than DNA and has about the same mobility. The table entries are sorted by effective mobility to highlight the appropriateness of the Tris/ $\mathrm{HCl}$ and $\mathrm{Hepes} / \mathrm{NaOH}$ as the LE and TE.

Table 1 The effective mobilities and minimum pH for several species of particular interest to this thesis, and to other common ITP experiments.

\begin{tabular}{|c|c|c|}
\hline Ionic Species & Effective Mobility $\left(\frac{u m / s}{V / m m}\right)$ & Minimum pH \\
\hline MS2 Bacteriophage & -7 & 7 \\
\hline Hepes(100mM) ${ }^{10}$ & -7.80 & 7.4 \\
\hline Hepes(10mM)10 & -10.15 & 7.4 \\
\hline DNA,RNA & -31.9 & 7.6 \\
\hline Fluorescein & -36 & 5 \\
\hline Alexa Fluor 488 & -47.2 & -2 \\
\hline Chloride & -79.1 & \\
\hline
\end{tabular}




\section{Theory of Operation}

This chapter theoretically derives the parameters of a transverse ITP device which result in the $\mathrm{x}$-axis being a projection of the time evolution of a traditional capillary ITP. The general geometry of the device is as shown in Figure 3, but specific parameters are left in symbolic form for usability in design.

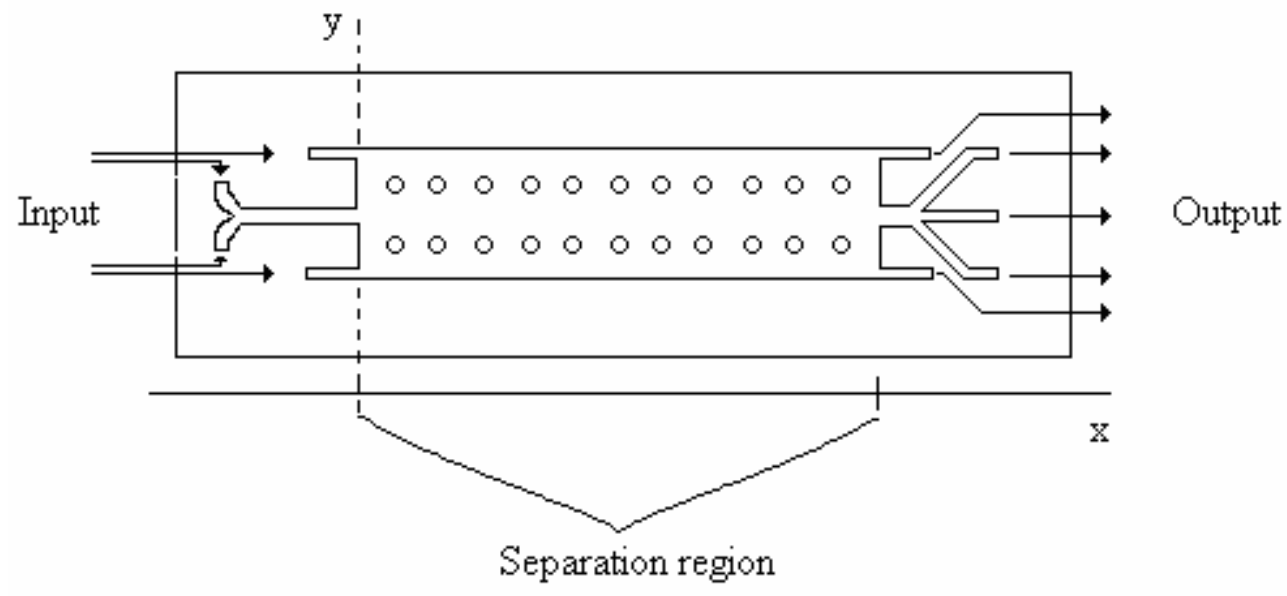

Figure 3. The coordinate system to be used in the development of a transverse ITP model. The $x$-axis is the direction of bulk fluid flow, and the $y$-axis is the direction of electrophoretic movement. The parallel rows of circles are the posts which prevent the channel from collapsing.

\subsection{Ion Transport}

The ion concentration everywhere in the separation channel is described by the Reynolds Transport Theorem, written here as

$$
\frac{\partial}{\partial t} \rho_{i}+\bar{\nabla} \bullet\left(\rho_{i} v_{i} \vec{E}\right)+\bar{\nabla} \bullet\left(\rho_{i} \vec{v}\right)-\bar{\nabla} \bullet\left(D_{i} \bar{\nabla} \rho_{i}\right)=0
$$


Equation 3.1 is derived in the Appendix. In all future instances of this equation, we assume that there is no variation of any quantity in the z-direction, and that within the separation channel, $D_{i}$ and $v_{i}$ are constant.

$$
\left.\frac{\partial}{\partial t} \rho_{i}+v_{i} \bar{\nabla} \bullet\left(\rho_{i} \vec{E}\right)+\bar{\nabla} \bullet\left(\rho_{i} \vec{v}\right)-D_{i} \nabla^{2} \rho_{i}\right)=0
$$

In a single spatial dimension with no bulk fluid flow, as is the case with capillary ITP, equation 3.1 becomes

$$
\frac{\partial}{\partial t} \rho_{i}+v_{i} \frac{\partial}{\partial y}\left(\rho_{i} E\right)-D_{i} \frac{\partial^{2}}{\partial y^{2}} \rho_{i}=0
$$

It will be a primary goal of this chapter to justify the reduction of equation 3.2 into a form similar to that of equation 3.3, and to establish the necessary conditions under which such a reduction is valid.

\subsection{Microfluidics aspect}

This section establishes the criteria necessary to ensure that turbulent flow is not present within the separation chamber, that diffusion along the direction of pressuredriven flow is negligible, and that no mixing of the LE and TE occurs prior to entering the separation channel. 


\subsubsection{Reynolds Number}

Equation 2.1 is reproduced here.

$$
\operatorname{Re}=\frac{\rho U_{0} L_{0}}{\eta}
$$

The target throughput is $100 \mu \mathrm{L} / \mathrm{min}$. With a separation channel cross-sectional area of $30,000 \mu \mathrm{m}^{2}$, the bulk velocity is $55,000 \mu \mathrm{m}^{2} / \mathrm{s}$. Using a characteristic length of $500 \mathrm{um}$ (the channel width), and the density and shear viscosity of water, the Reynolds number for the separation channel is 27.5 , which is near enough to the negligent-turbulent-flow operating region that turbulent flow is not considered in future analysis.

\subsubsection{Péclet Number}

Equation (2.2) is reproduced here.

$$
P e=\frac{U_{0} L_{0}}{D}
$$

Using the same characteristic lengths and velocities as for the Reynolds Number, and a diffusion coefficient of $2000 \mu \mathrm{m}^{2} / \mathrm{s}$ for the smallest particles (chloride), the Péclet number within the separation channel is 13,750 , which is well into the negligible-diffusion region. Note that the bulk velocity is entirely horizontal while the diffusion of interest would happen perpendicular to that flow. For this reason, the horizontal diffusion component is discarded in future analysis, but the vertical component remains. 
The sample inlet is narrower than the separation channel, necessitating a much higher bulk velocity. This effectively reduces the pre-separation mixing time enough that the initial condition of a step-function analyte concentration is preserved until injection into the separation region.

\subsection{Electrical aspect}

This section contains the electrical analysis for transverse ITP, including the highest-level discrete component circuit, and the continuum analysis within the separation channel.

\subsubsection{Circuit diagram}

Figure 4 is an electrical model of the entire system. It is a straightforward system with the exception of what goes on inside the separation channel. This is why the separation channel has most of a chapter written about it, and the rest of it gets only these words: voltage divider.

Typical values for the resistance of the resistances are $100 \mathrm{k} \Omega$ for the 6 "tubing and $200 \mathrm{k} \Omega$ for the inlets and outlets. These are based off of a $30 \mathrm{mS} / \mathrm{cm}$ solution (1M buffers) within these tubes. The separation channel resistance is typically $20 \mathrm{k} \Omega$ or less, depending on the conductivity of the sample. 


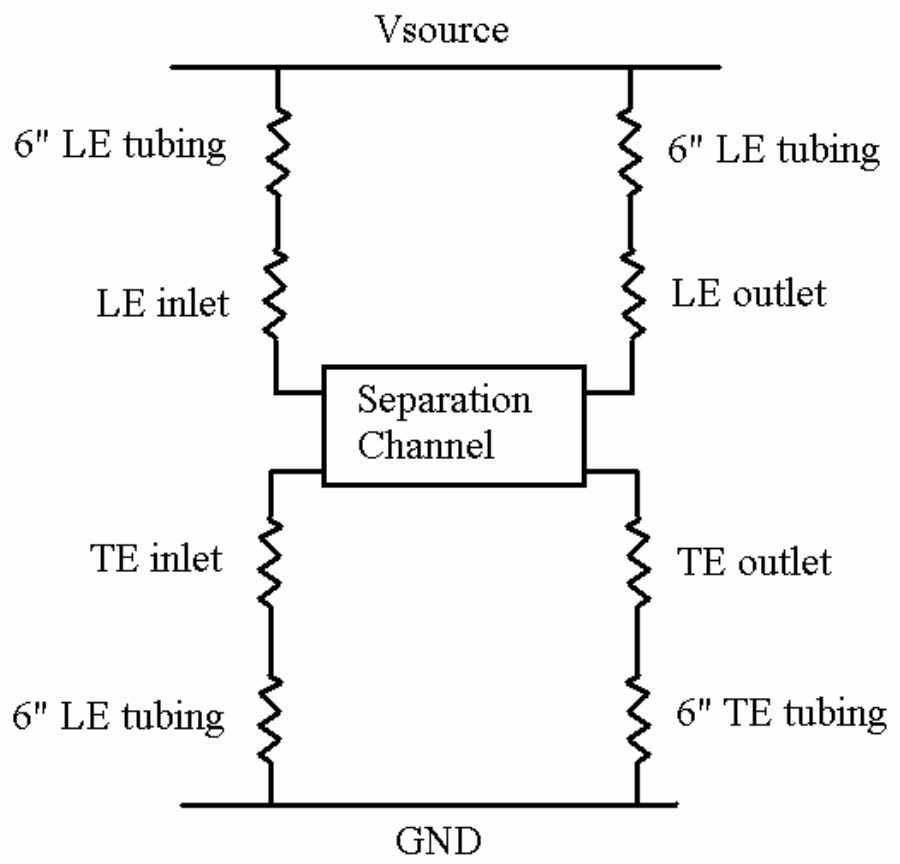

Figure 4. Circuit-element representation of the ITP system. The voltage drop across the inlet/outlet channels and tubing account for up to $90 \%$ of the total applied voltage in some cases.

\subsubsection{Electric field for low conductivity sample}

This section aims to establish that the Electric field in the sample channel is uniform and vertical. If the Liquid Electrode streams are shown to be equipotential surfaces, then the field between them must be uniform. Figure 5 shows the coordinate system and variables used in the analysis, while Figure 6 shows an estimation of the electric field within the various regions of the chip. 


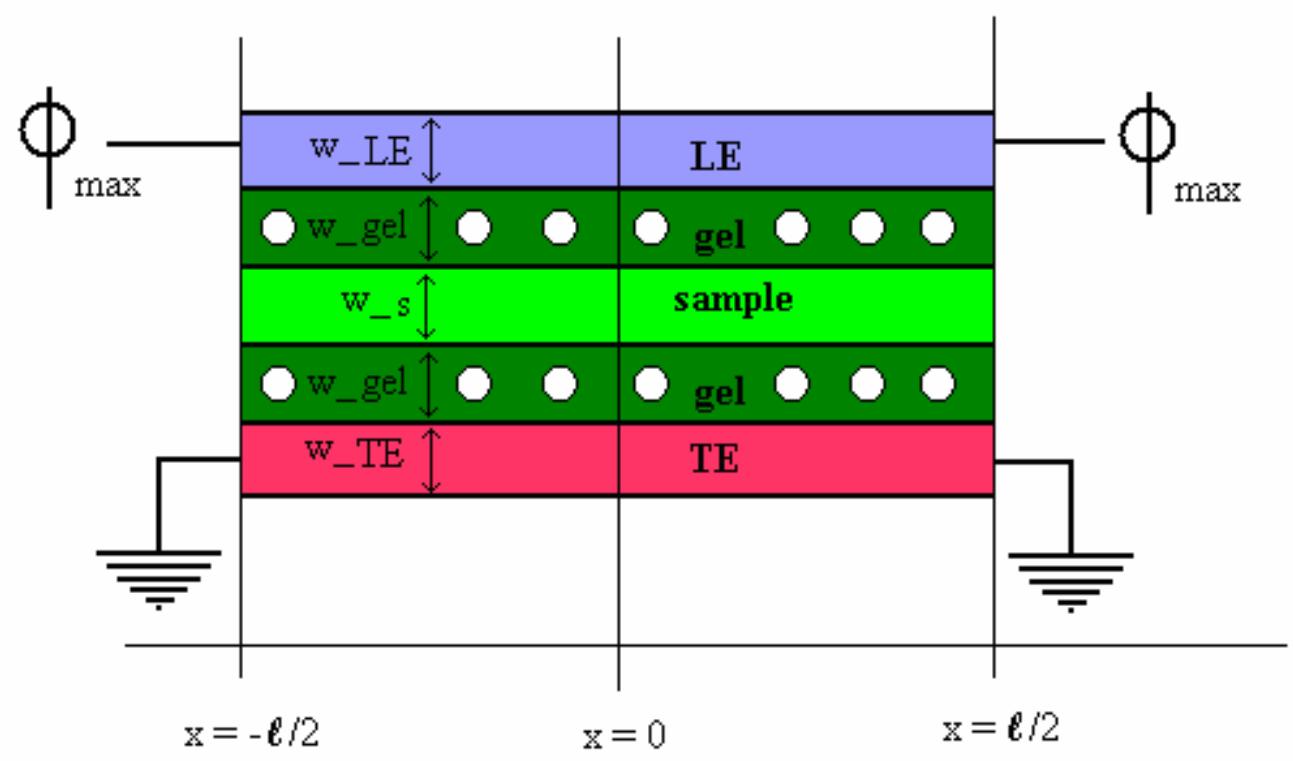

Figure 5. The width of the LE, TE, gel, and sample regions are defined, as well as the coordinate system used in the derivation of the electric field in the LE region. The sample in this case is pink, and the white "holes" are the posts which keep the 2 glass surfaces from collapsing.

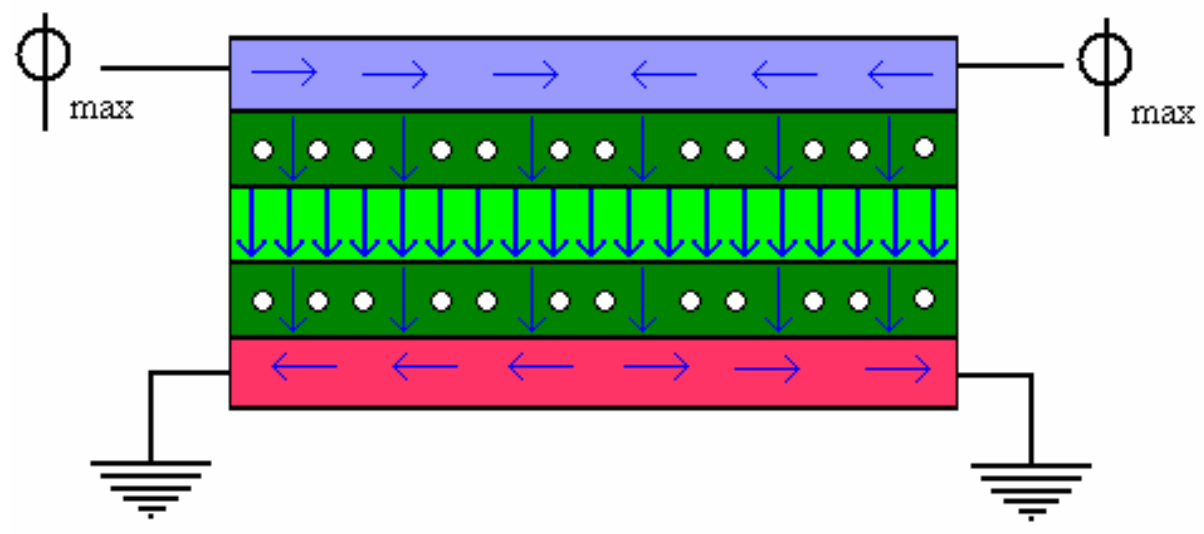

Figure 6. The electric field present in the chip. Darker, more densely packed arrows indicate that the field is stronger in such regions. The sample in this case is pink, and the white "holes" are the posts which keep the 2 glass surfaces from collapsing. 
We assume that the sample conductivity is much less than the LE,TE, and gel conductivities.

$$
\sigma_{s}<<\sigma_{g e l} \approx \sigma_{L E}=\sigma_{T E}
$$

We also assume that LE, gel, and sample regions are all of comparable width.

$$
w_{s} \approx w_{g e l} \approx w_{L E} \approx w_{T E}
$$

Beginning with Faraday's Law, we note that in the steady state, nothing varies with time, magnetic field included.

$$
\bar{\nabla} x \bar{E}=-\frac{d \bar{B}}{d t}=0
$$

This implies that the electric field is in fact the gradient of the potential.

$$
\bar{E}=\frac{\bar{J}}{\sigma}=-\bar{\nabla} \varphi
$$

We make the assumption that there is no significant variation in $\varphi_{L E}$ in the y direction.

This assumption will be validated later.

$$
\frac{d \varphi_{L E}}{d x}=-\frac{J_{x L E}}{\sigma_{L E}}
$$

Applying the discrete version of KCL to Figure 7 yields

$$
\left[J_{x}(x+\Delta x)-J_{x}(x)\right] w_{L E}=-J_{\text {yavg }} \Delta x
$$




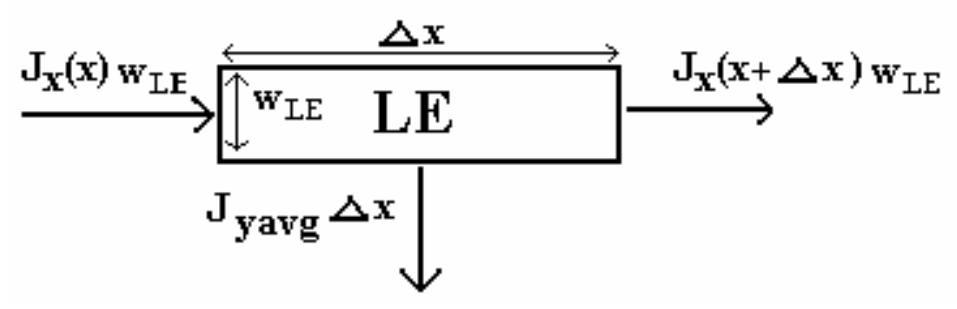

Figure 7. Differential element of the high-conductivity (undiluted $1 \mathrm{M}$ buffer) LE stream.

Some quick algebra and the introduction of a limit yields

$$
\lim _{\Delta x \rightarrow 0} \frac{\left[J_{x L E}(x+\Delta x)-J_{x L E}(x)\right]}{\Delta x}=-\lim _{\Delta x \rightarrow 0} \frac{J_{y a v g}}{w_{L E}}
$$

From the definition of the derivative, this simplifies to

$$
\frac{d J_{x L E}}{d x}=-\frac{J_{y}(x)}{w_{L E}}
$$

By Ohm's Law, applied to an infinitesimally narrow strip connecting the LE and TE streams

$$
J_{y}(x)=\frac{\varphi_{L E}(x)-\varphi_{T E}(x)}{\frac{w_{s}}{\sigma_{s}}+\frac{2 w_{g e l}}{\sigma_{g e l}}}
$$

By symmetry

$$
\varphi_{L E}(x)+\varphi_{T E}(x)=\varphi_{\max }
$$

Approximate using equations (3.4) and (3.5)

$$
\frac{w_{s}}{\sigma_{s}}+\frac{2 w_{g e l}}{\sigma_{g e l}} \approx \frac{w_{s}}{\sigma_{s}}
$$


Substituting equations (3.13) and (3.14) into (3.12) yields

$$
J_{y}(x)=\left(2 \varphi_{L E}(x)-\varphi_{\max }\right) \frac{\sigma_{s}}{w_{s}}
$$

Differentiating equation (3.8) and substituting equation (3.11) yields

$$
\frac{d^{2} \varphi_{L E}}{d x^{2}}=-\frac{1}{\sigma_{L E}} \frac{d J_{x L E}}{d x}=\frac{1}{\sigma_{L E}} \frac{J_{y}(x)}{w_{L E}}
$$

Substitute equation (3.15) into (3.16) yields

$$
\frac{d^{2} \varphi_{L E}}{d x^{2}}=\left(2 \varphi_{L E}(x)-\varphi_{\max }\right) \frac{\sigma_{s}}{\sigma_{L E}} \frac{1}{w_{s} w_{L E}}
$$

Solving this linear second order homogenous ODE gives

$$
\varphi_{L E}(x)=A \cosh (\sqrt{2 m} x)+\frac{\varphi_{\max }}{2}
$$

Where

$$
m=\frac{\sigma_{s}}{\sigma_{L E}} \frac{1}{w_{s} w_{L E}}
$$

And $A$ is a constant such that $\varphi_{L E}(-l / 2)=\varphi_{\max }$

$\varphi_{L E}(x)$ is plotted for a range of values of $\frac{\sigma_{s}}{\sigma_{L E}}$ for two extreme cases: $l \approx 30 w_{s}$ (Figure 8) and and $l \approx 4 w_{s}$ (Figure 9). 


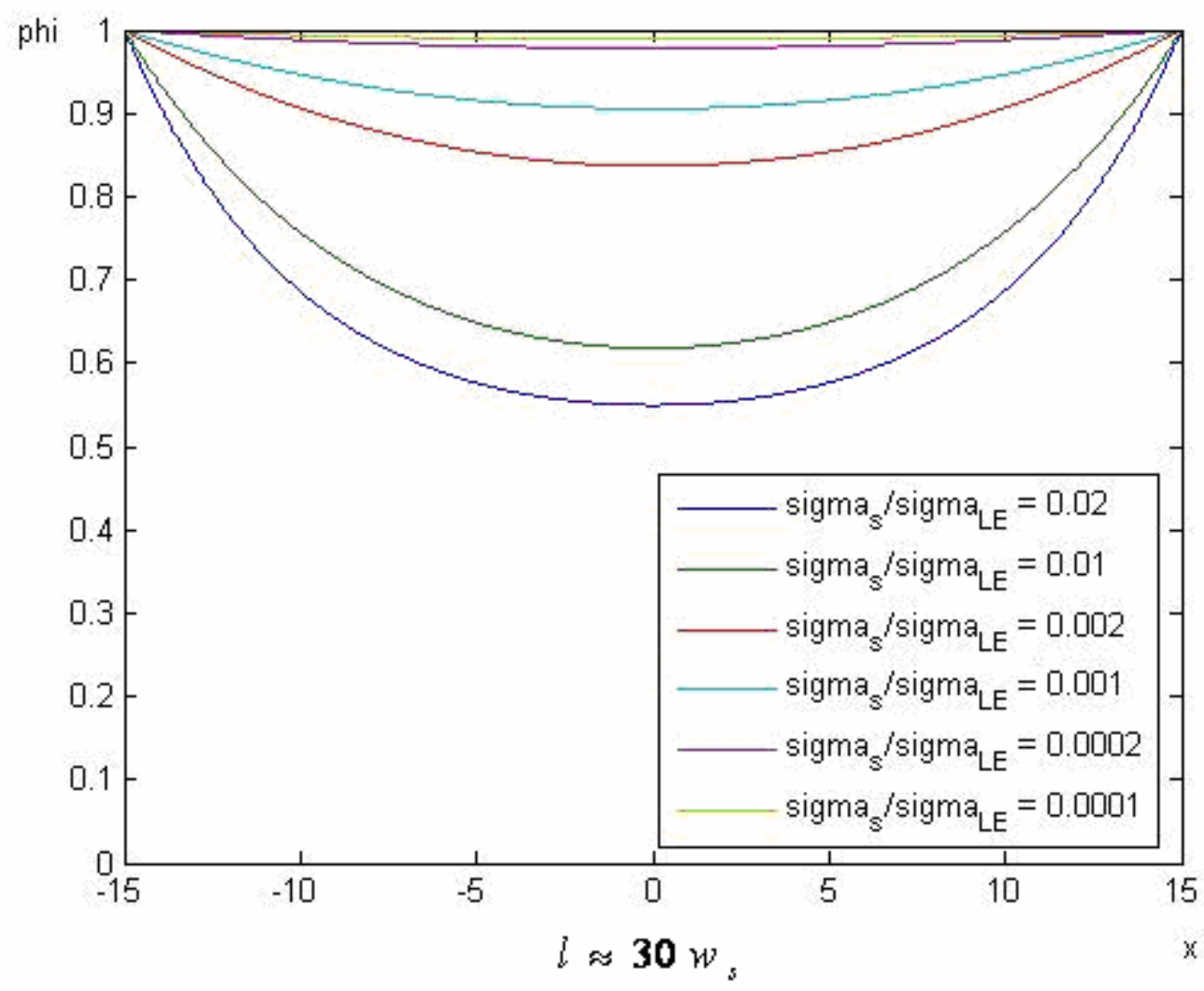

Figure 8. The magnitude of the electric potential along the longest LE channel. The sag in the potential is considered extreme in cases where the conductivity ratios is greater than 0.001 . 


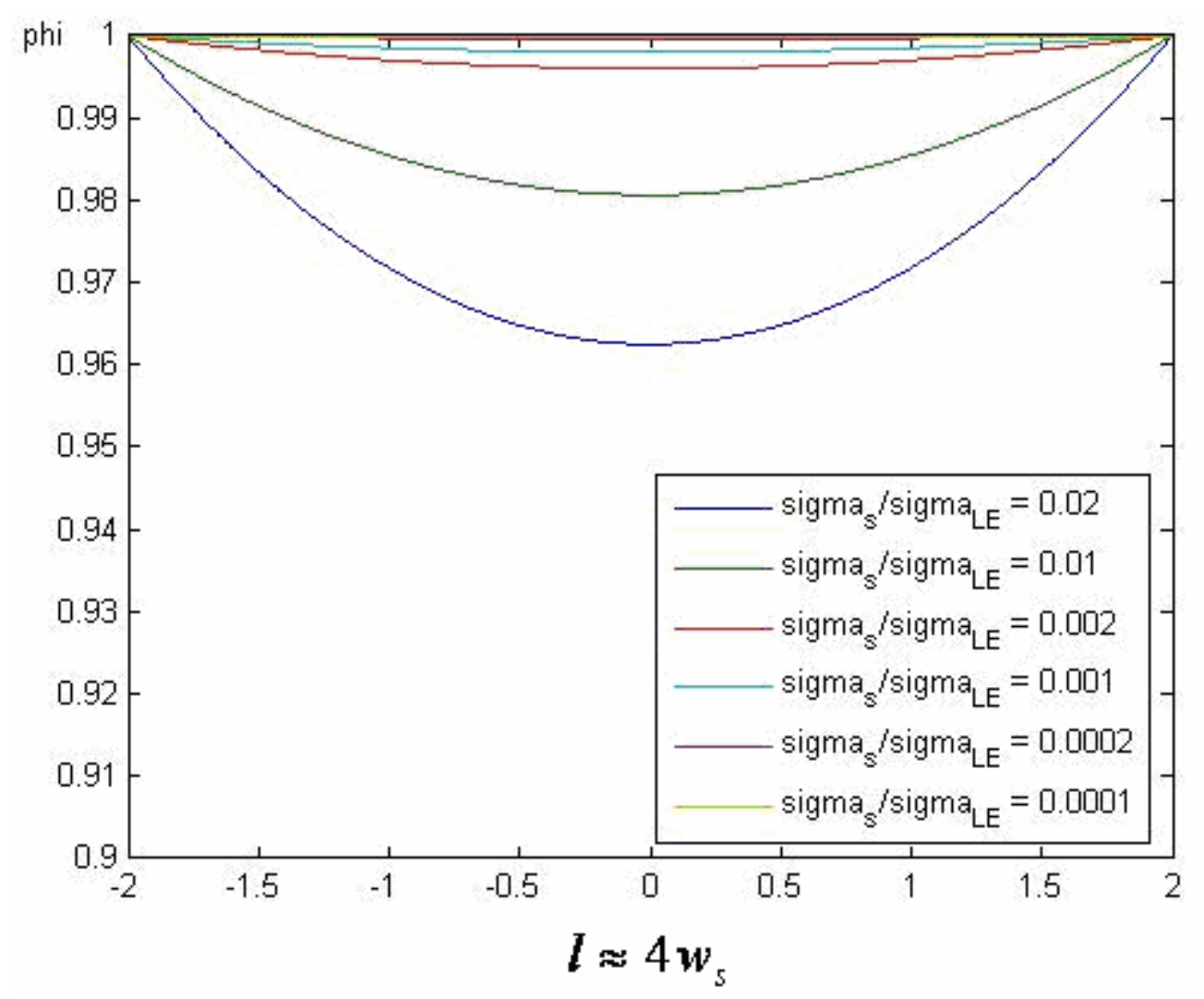

Figure 9. The magnitude of the electric potential along the shortest LE channel. Note the change of scale. For this case, conductivity ratios as low as 0.02 still yield an approximately equipotential surface.

With the shorter channel, the cosh function is well approximated as a constant given that $\sigma_{s} \leq 0.02 \sigma_{L E}$, and the liquid electrodes can be justly described as equipotential surfaces, resulting in a uniform, vertical electric field in the sample region. The lack of significant variation of the potential along the longest axis of the liquid electrodes justifies the assumption that there was no significant variation along the shorter axis.

From Figure 8 and Figure 9 it is evident that the requirements on $\frac{\sigma_{s}}{\sigma_{L E}}$ are much more strict for the longer channel. It is necessary that $\sigma_{s} \leq 0.001 \sigma_{L E}$ to make the same claim of equipotentiality. The advantage of the longer channel, provided that the conductivity 
requirements can be met, is that a weaker electric field is required to achieve separation than would be necessary for the shorter channel.

From this analysis it is apparent that the effectiveness and efficiency of a highconductivity sample are greatly diminished, due to the complete non-uniformity of the electric field.

\subsection{Ion Transport Revisited}

Returning to Equation 3.1, this section aims to reduce the daunting general case into something solvable, or at least recognizable.

$$
\left.\frac{\partial}{\partial t} \rho_{i}+v_{i} \bar{\nabla} \bullet\left(\rho_{i} \vec{E}\right)+\bar{\nabla} \bullet\left(\rho_{i} \vec{v}\right)-D_{i} \nabla^{2} \rho_{i}\right)=0
$$

\subsubsection{Simplifications, justifications}

Here we abuse all the great results from previous sections to bust that pesky PDE down into something sexy and solvable.

1) The bulk fluid velocity is considered to be perfectly horizontal and constant everywhere. This is of course not true at places where the channel abruptly changes width, but is valid for the majority of the separation region. From the Reynolds number calculation in section 3.2.1 it is evident that the presence of turbulent flow is non- 
existent, leaving a laminar flow driven by a pressure difference between separation channel inlet and outlet. This is expressed as $\vec{v}=v \hat{x}$, and reduces Equation 3.2 to

$$
\frac{\partial}{\partial t} \rho_{i}+v_{i} \bar{\nabla} \bullet\left(\rho_{i} \vec{E}\right)+v \frac{d}{d x} \rho_{i}-D_{i} \nabla^{2} \rho_{i}=0
$$

2) Diffusion in the $x$ direction is negligible. From the Péclet number calculation in section 3.2.2 it is evident that given the significant horizontal velocity of the fluid, ion transport in this direction via diffusion is negligible. The presence of an $\mathrm{x}$-direction advection term in Equation 3.19 allows us to discard horizontal diffusion in all future considerations. Equation 3.19 now reduces to

$$
\frac{\partial}{\partial t} \rho_{i}+v_{i} \bar{\nabla} \bullet\left(\rho_{i} \vec{E}\right)+v \frac{d}{d x} \rho_{i}-D_{i} \frac{\partial^{2}}{d y^{2}} \rho_{i}=0
$$

3) The electric field exists entirely in the y direction. From the result of section 3.3.2, within the constraints given in that section, the electric field may be approximated as $\vec{E}=E \hat{y}$. Equation 3.20 now reduces to

$$
\frac{\partial}{\partial t} \rho_{i}+v_{i} \frac{d}{d y}\left(\rho_{i} E\right)+v \frac{d}{d x} \rho_{i}-D_{i} \frac{\partial^{2}}{d y^{2}} \rho_{i}=0
$$


4) The final simplification is the outright declaration that I am only interested in the steady-state solution. The start up transients exist for only the blink of an eye in the lifetime of a single sample processing period (several minutes, perhaps hours). This is manifest as $\frac{d}{d t} \rho_{i}=0$ which, along with the rearrangement of the other terms, reduces equation (3.21) to

$$
v \frac{d}{d x} \rho_{i}+v_{i} \frac{d}{d y}\left(\rho_{i} E\right)-D_{i} \frac{\partial^{2}}{d y^{2}} \rho_{i}=0
$$

From comparison to equation 3.3, reprinted here, it is evident that the transverse ITP system is equivalent to a time-scale projection of capillary ITP, under the coordinate transformation $x=v t$.

$$
\frac{\partial}{\partial t} \rho_{i}+v_{i} \frac{\partial}{\partial y}\left(\rho_{i} E\right)-D_{i} \frac{\partial^{2}}{\partial y^{2}} \rho_{i}=0
$$

\subsubsection{Solution}

There are three charged species of relevance in ITP. These are the LE, TE, and analyte. Typically, the initial analyte concentration is several orders of magnitude less than that of the TE, and thus contributes little to the conductivity of the TE zone where it 
initially resides. It is therefore sufficient to first characterize the electric field as if the analyte were not present, and then characterize analyte movement in such a field. Figure 10 shows the initial LE, TE, and analyte distributions for the following analysis.
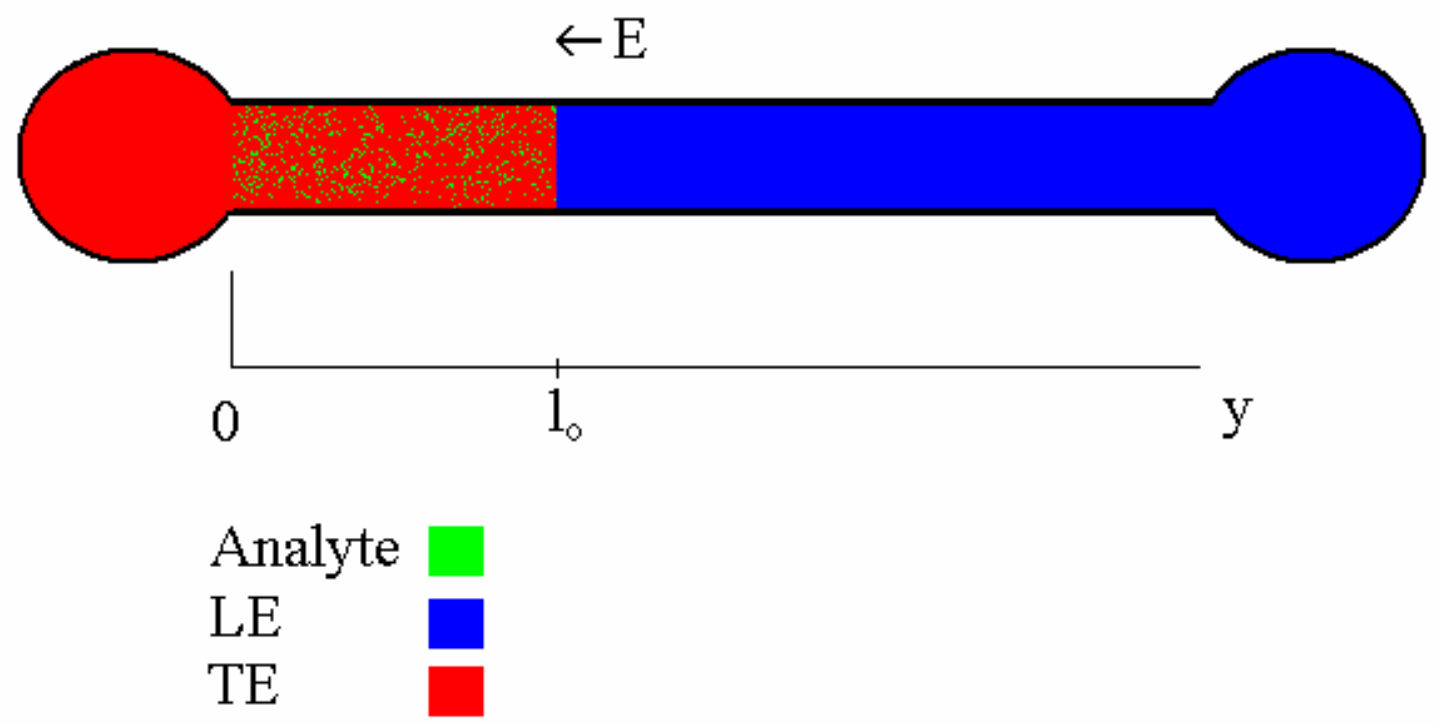

Figure 10. The coordinate system to be used in the following analysis. The above frame corresponds to $t=0$.

We see in capillary ITP that after separation has occurred, the homogeneous LE and TE zones travel down the capillary at the same speed.

$$
\begin{array}{r}
v_{T E}=v_{L E} \\
E_{T E} v_{T E}=E_{L E} v_{L E}
\end{array}
$$

KVL imposes the additional condition 


$$
E_{T E} l_{T E}+E_{L E} l_{L E}=\phi_{\max }
$$

Eq. 3 can be combined with Eq. 2 to yield

$$
E_{T E}\left(l_{L E} \frac{v_{T E}}{v_{L E}}+l_{T E}\right)=\varphi_{\max }
$$

The conditions of the ITP can be controlled such that $\frac{v_{T E}}{v_{L E}}<<1$, and $l_{L E} \approx l_{T E}$, so that

(3.26) can be approximated as

$$
E_{T E}=\frac{\varphi_{\max }}{l_{T E}}
$$

With the coordinate origin at the mouth of the TE reservoir, the coordinate of the boundary is the length of the TE region. The speed with which the boundary moves is thus

$$
\frac{d}{d t} l_{T E}=E_{T E} v_{T E}
$$

Using the relation in (3.27), this can be rewritten as

$$
\frac{d}{d t} l_{T E}=\frac{\varphi_{\max } v_{T E}}{l_{T E}}
$$

And solved for $l_{T E}$ to give

$$
l_{T E}(t)=\sqrt{2 \phi_{\max } v_{T E} t+l_{0}^{2}}
$$


Where $l_{0}$ is the initial length of the TE region. The separation will be considered complete when all of the analyte has migrated from the bottom of the TE region to the boundary. The distance which the analyte has traveled is

$$
d_{A n}(t)=\int_{0}^{t} v_{A n}(\tau) d \tau=\int_{0}^{t} v_{A n} E_{T E}(\tau) d \tau
$$

Using (3.27) and (3.30), this becomes

$$
d_{A n}(t)=\int_{0}^{t} v_{A n} \frac{\varphi_{\max }}{\sqrt{2 \phi_{\max } v_{T E} \tau+l_{0}^{2}}} d \tau
$$

Which, after integration, simplifies to

$$
d_{A n}(t)=\frac{v_{A n}}{v_{T E}}\left(\sqrt{2 \phi_{\max } v_{T E} t+l_{0}^{2}}-l_{0}\right)
$$

It is convenient at this point to introduce the dimensionless number $\gamma$ as

$$
\gamma=\frac{v_{A n}}{v_{T E}}
$$

Setting (3.33) and (3.30) equal to each other, and exploiting (3.34) gives

$$
\gamma \sqrt{2 \phi_{\max } v_{T E} t_{s}+l_{0}^{2}}-\lambda_{0}=\sqrt{2 \phi_{\max } v_{T E} t_{s}+l_{0}^{2}}
$$

Several steps of basic algebra reduce this to

$$
t_{s}=\frac{\gamma(2 \gamma-1)}{(\gamma-1)^{2}} \frac{l_{0}^{2}}{2 \phi_{\max } v_{A n}}
$$


Equation 3.36 is the necessary time for separation, after which the analyte remains separated until the LE reservoir is reached. From this we see that the time required for separation is inversely proportional to the applied voltage. The position of the boundary at the first moment of full separation is given by substituting (3.36) into (3.30), which simplifies substantially to become

$$
l_{s_{-} \min }=l_{T E}\left(t_{s}\right)=l_{0} \frac{\gamma}{\gamma-1}
$$

And so we see that unlike the necessary separation time, the position of the focused band is entirely unrelated to the applied voltage.

The analyte is of course not infinitely concentrated at the boundary. Diffusion will cause it to disperse to some extent. To determine this extent, return to Equation 3.3.

$$
\frac{\partial}{\partial t} \rho_{i}+v_{i} \frac{\partial}{\partial y}\left(\rho_{i} E\right)-D_{i} \frac{\partial^{2}}{\partial y^{2}} \rho_{i}=0
$$

It is now advantageous to shift to a different coordinate system, whose origin is centered at the boundary and moves with it. This is expressed mathematically by the transformation

$$
q=y-v t-l_{s_{-} \min }
$$


where $v=v_{T E} E_{T E}$. Rewriting Equation 3.3e, using the chain rule to replace the spatial and time derivatives in terms of $\mathrm{q}$, and recognizing also that the electric field is piece-wise constant results in the ordinary differential equation

$$
\frac{d^{2}}{d q^{2}} \rho_{A n}+\left(\frac{v_{T E} E_{T E}-v_{A n} E}{D_{A n}}\right) \frac{d}{d q} \rho_{A n}=0
$$

In the TE region $(q \leq 0)$ this is easily solved as

$$
\rho_{A n}(q)=\rho_{0} e^{\left(\frac{v_{A n} E_{T E}-U_{T E} E_{T E}}{D_{A n}}\right) q}
$$

And in the LE region $(q \geq 0)$, where $E \approx 0$ as

$$
\rho_{A n}(q)=\rho_{0} e^{\frac{-v_{T E} E_{T E}}{D_{A n}} q}
$$

where $\rho_{0}$ is an unknown constant. $\rho_{0}$ can be found by integrating the entire focused region, and setting this quantity equal to the total amount of analyte in solution.

$$
\int_{-\infty}^{+\infty} \rho_{A n}(y) d y=l_{o} \rho_{u}
$$

where $\rho_{u}$ is the concentration in the un-focused state, prior to the start of the ITP. Splitting the integral into separate LE and TE integrals gives

$$
\frac{\rho_{0} D_{A n}}{v_{A n} E_{T E}-v_{T E} E_{T E}}+\frac{\rho_{0} D_{A n}}{v_{T E} E_{T E}}=l_{o} \rho_{u}
$$

which is solved to give a maximum concentration of 


$$
\rho_{0}=\frac{l_{o} \rho_{u}}{D_{A n}} \frac{\left(v_{A n}-v_{T E}\right)}{\gamma} \frac{\phi_{\max }}{l_{T E}}
$$

where Equation 3.27 has been used to establish the dependence upon $\phi_{\max }$. Figure 11 illustrates the effect of $\gamma$ on the concentration profile of the analyte, for given values of. $l_{o}, \rho_{u}, \phi_{\max }, l_{T E}, D_{A n}, v_{A n}$

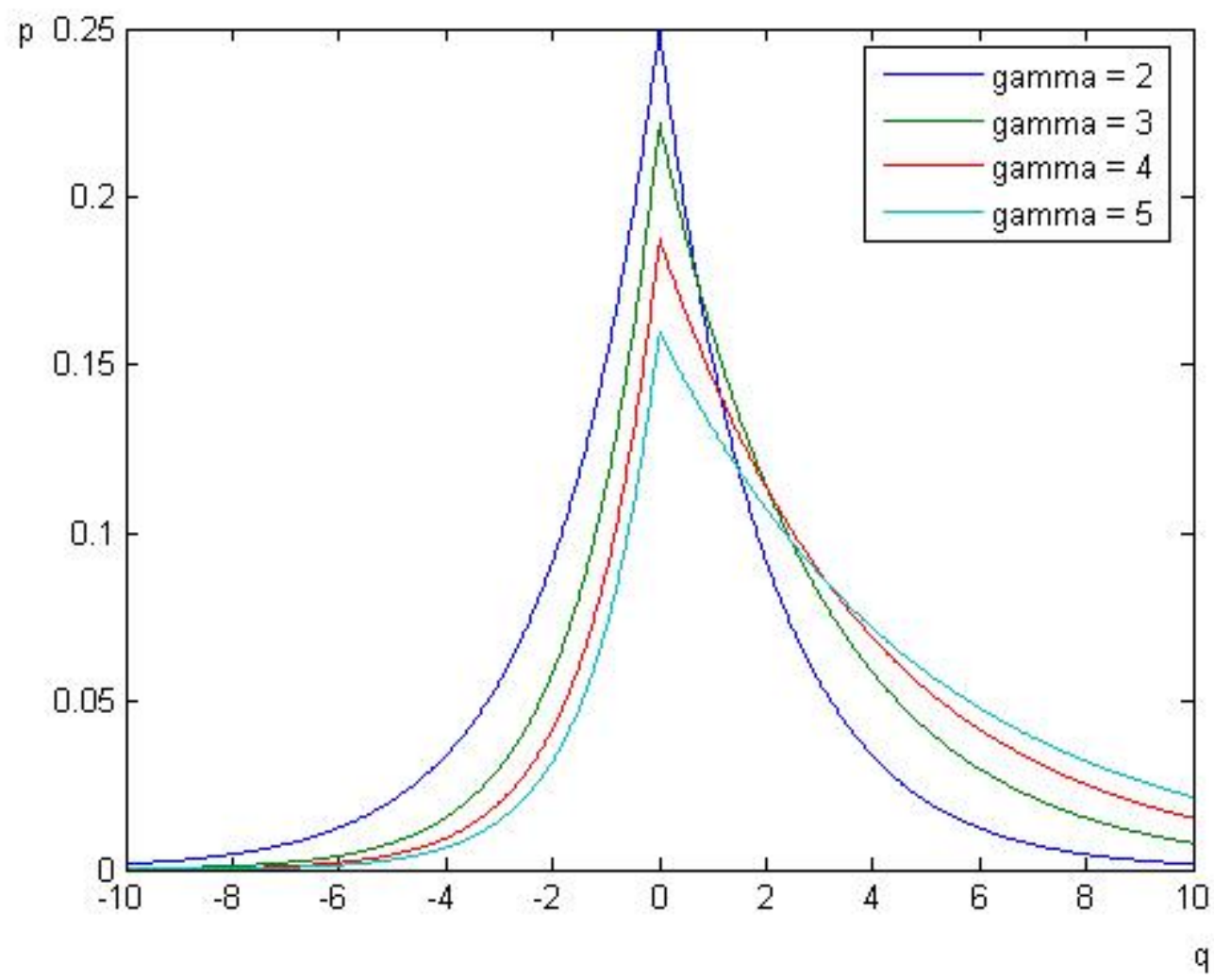

Figure 11. Concentration profile of the Analyte with all equal to 1 . It is seen that with increasing the peak concentration decreases and the analyte profile becomes more spread out. At the profile is symmetric about.

I will define $l_{A n_{-} T E}$ and $l_{A n_{-} L E}$ as the edges of the analyte band. I also define $r$ as the fraction of the original sample which resides within the band. The band edges are thus 


$$
\begin{gathered}
l_{A n_{-} T E}=\frac{\ln (1-r)}{-\left(\frac{v_{A n} E_{T E}-v_{T E} E_{T E}}{D_{A n}}\right)}=\frac{\ln \left(\frac{1}{1-r}\right) D_{A n}}{v_{A n}-v_{T E}} \frac{l_{T E}}{\phi_{\max }} \\
l_{A n_{-} L E}=\frac{\ln (1-r)}{-\left(\frac{v_{T E} E_{T E}}{D_{A n}}\right)}=\frac{\ln \left(\frac{1}{1-r}\right) D_{A n}}{v_{T E}} \frac{l_{T E}}{\phi_{\max }}
\end{gathered}
$$

The total length of the analyte region is then the sum of $l_{A n_{-} T E}$ and $l_{A n_{-} L E}$.

$$
l_{A n}=\frac{\ln \left(\frac{1}{1-r}\right) D_{A n}}{v_{A n}-v_{T E}} \frac{v_{A n}}{v_{T E}} \frac{l_{T E}}{\phi_{\max }}=\ln \left(\frac{1}{1-r}\right) \frac{D_{A n}}{v_{A n}} \frac{l_{T E}}{\phi_{\max }} \frac{\gamma^{2}}{\gamma-1}
$$

In transverse ITP, the sample band is reclaimed through its own outlet, ideally of width $l_{A n}$. We expect that the contents of the outlet will re-homogenize in the collection vessel. In this case, the net increase in concentration is

$$
C=r \frac{l_{0}}{l_{A n}}=\frac{r}{\ln \left(\frac{1}{1-r}\right)} \frac{v_{A n}}{D_{A n}} \frac{\phi_{\max }}{l_{T E}} \frac{\gamma-1}{\gamma^{2}} l_{0}
$$

Equations 47 and 48 have several important and interesting implications. The most useful of these is the dependence on $\phi_{\max }$, which can be used to directly reduce the analyte band length, and thus increases the concentration of the recovered sample stream. The next useful implication is the dependence on $l_{T E}$, which introduces time dependence. As the 
band propagates down the capillary, the sample becomes less concentrated, which suggests that there is a penalty for hesitating to reclaim the sample.

The role of gamma is not so clear, due to its appearance in both numerator and denominator. For a given $l_{T E}, l_{A n}$ is minimized and $C$ is maximized when $\gamma=2$, which demands a minimum channel length of $2 l_{0}$ for full separation (Equation 15). An interesting effect of this is that $\gamma=2$ results in a perfectly symmetric analyte band (found by comparing Equations 3.45 and 3.46 with the condition $\left.v_{A n}=2 v_{T E}\right)$. If $l_{T E}$ is not fixed, but collection occurs at the optimal moment, $t_{s}$, when $l_{T E}$ is at the minimum necessary length for full separation (Equation 3.15), Equation 48 becomes

$$
C=\frac{r}{\ln \left(\frac{1}{1-r}\right)} \frac{v_{A n}}{D_{A n}} \frac{(\gamma-1)^{2}}{\gamma^{3}} \phi_{\max }
$$

In this case, $\mathrm{C}$ is maximized when $\gamma=3$, requiring a minimum channel length of $\frac{3}{2} l_{0}$.

Not surprisingly, $C$ approaches its maximum value as $r$ approaches zero. This is the case of an infinitely narrow sample stream, centered on the LE/TE boundary where the concentration is at its maximum. $C$ approaches zero as $r$ approaches one, but this does not consider the finite limit imposed on $l_{A n}$ by the physical dimensions of the separation channel. 
After acceptable values for $r$ and $C$ are chosen, the minimum value of $\phi_{\max }$ is readily determined. Figure 12 illustrates the dependence of $C$ upon $r$ for given values of $v_{A n}, D_{A n}, \gamma, \varphi_{\max }$.

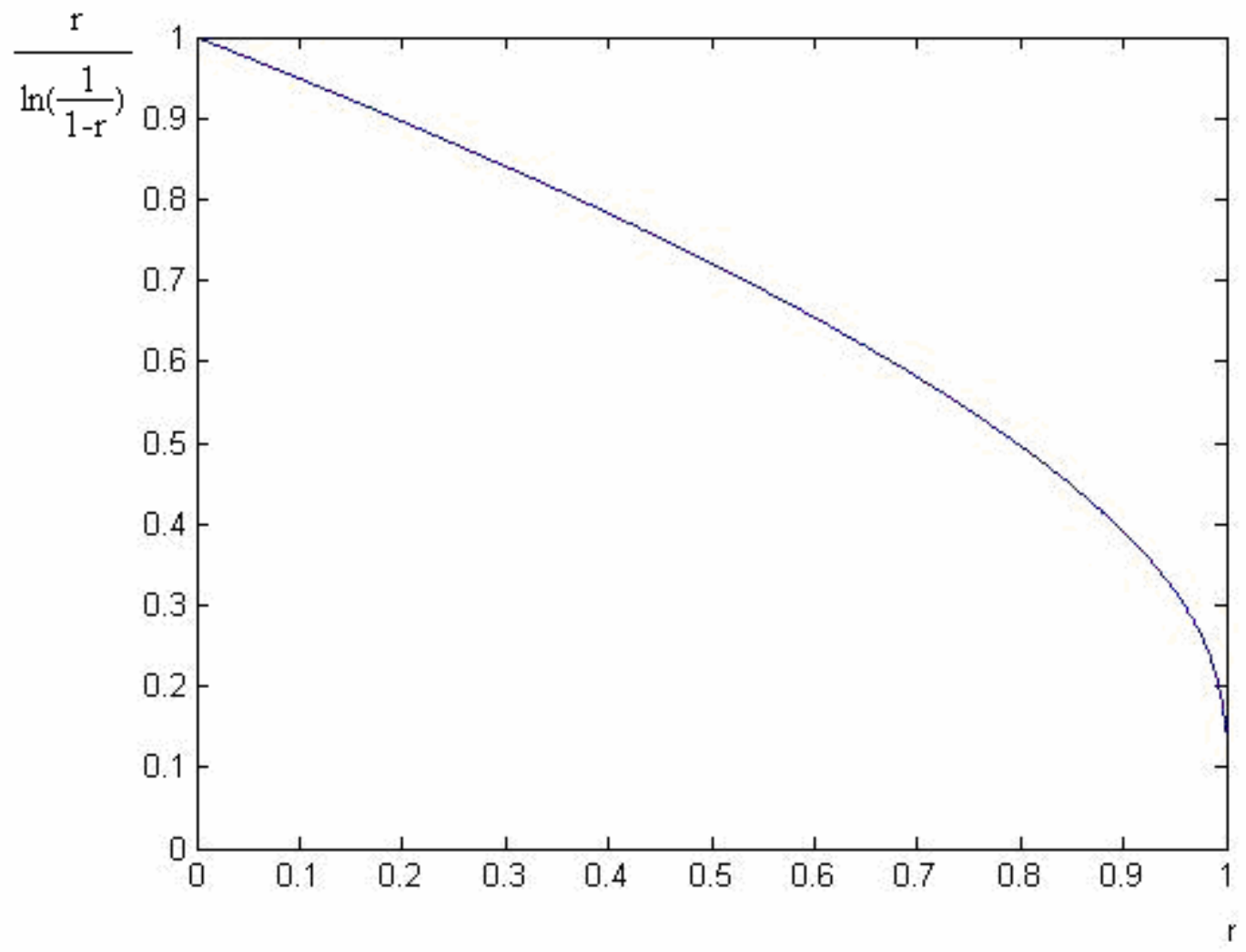

Figure 12. The dependence of $C$ upon $r$. In practice, when an acceptable $r$ is determined, the other parameters (mostly) can be manipulated to achieve the desired $C$.

Figure 13 shows the expected appearance of the time-smeared capillary-ITP, as dictated by the results of this section. 


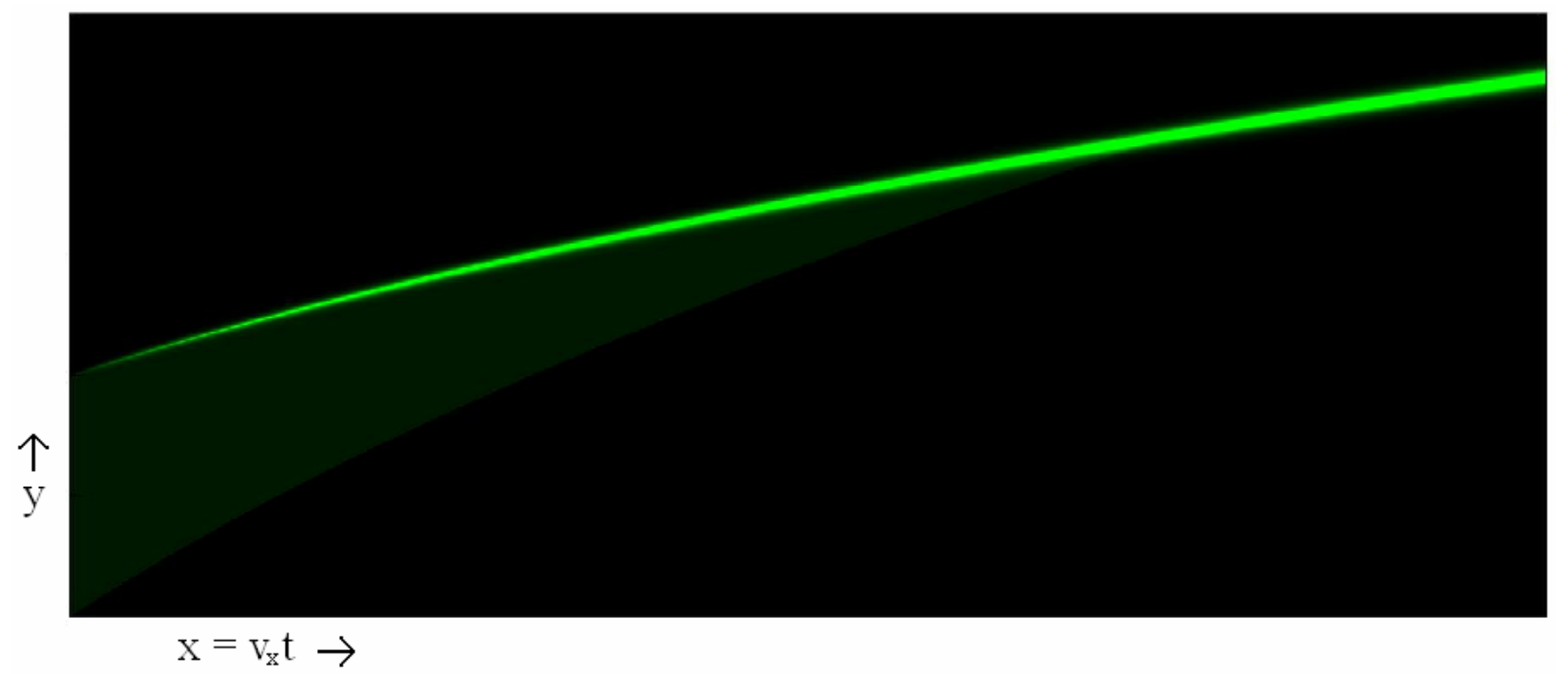

Figure 13. Time smear of the one-dimensional ITP, where ITP occurs on the $y$-axis and time is projected on the $x$-axis. This is what we expect to see when we observe the transverse ITP chip running. 


\section{Device Construction}

This chapter addresses the physical construction of the transverse-ITP test-setup at

LLNL. Commercially available paraphernalia such as syringes and plastic tubing are not

discussed. Figure 14 shows the entire experimental setup, minus sample illumination and microscope/camera.

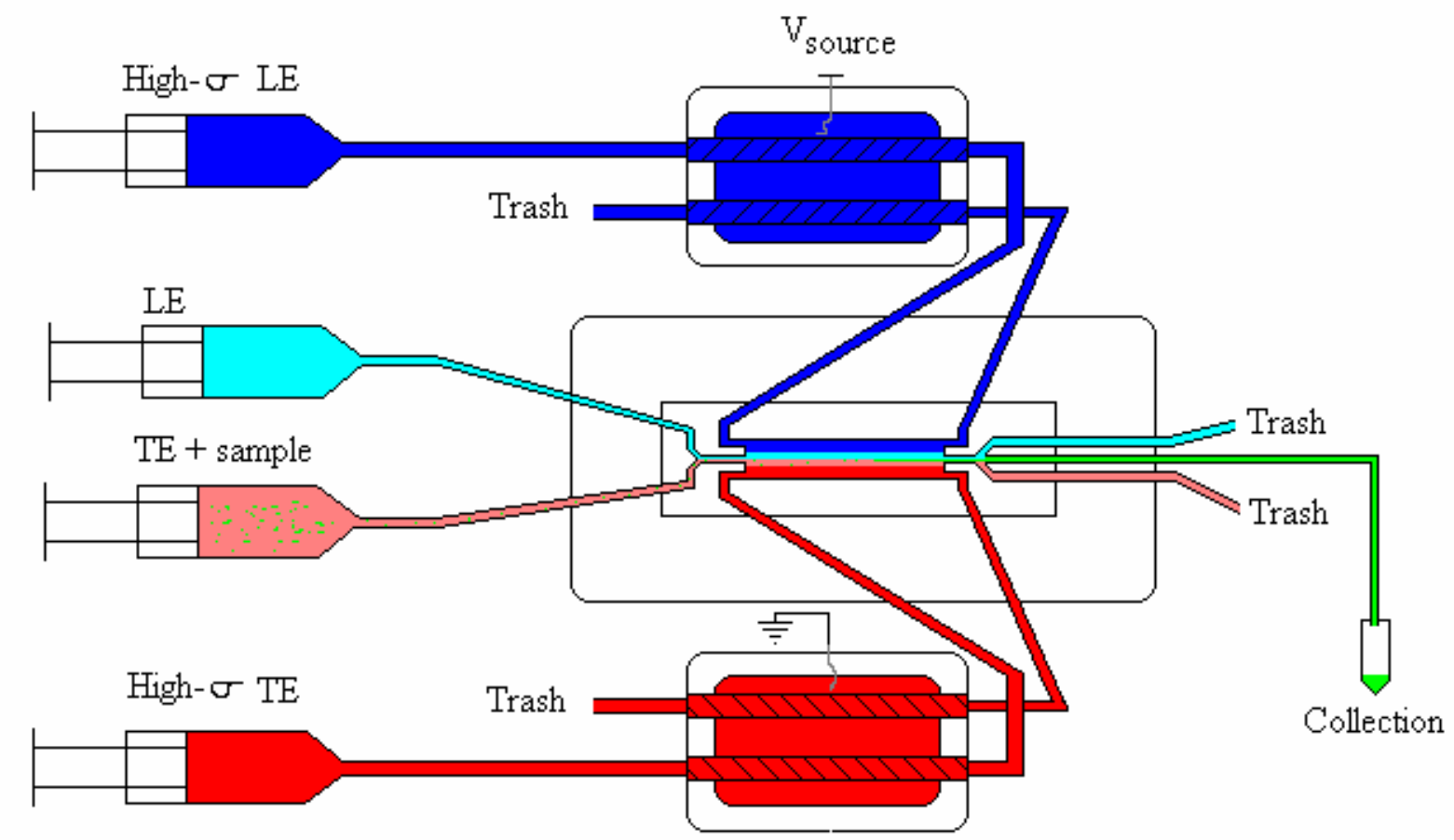

Figure 14. The entire experimental setup, including source syringes, salt bridges, separation channel, collection vials and all connective tubing. 


\subsection{Chip}

\subsubsection{Design}

There are four different chips using the same fundamental design, all shown in

Figure 15. The difference is in the separation channel length, which translates directly to residence time for a fixed sample flow rate. The four lengths are $3 \mathrm{~mm}, 13 \mathrm{~mm}, 23 \mathrm{~mm}$, and $33 \mathrm{~mm}$. For simplicity in construction and future interfacing with other fluidics equipment, the positions of the inlet and outlet holes are kept the same on each chip, with the inlet and outlet channels changing length to accommodate reduced or increased separation channel length.
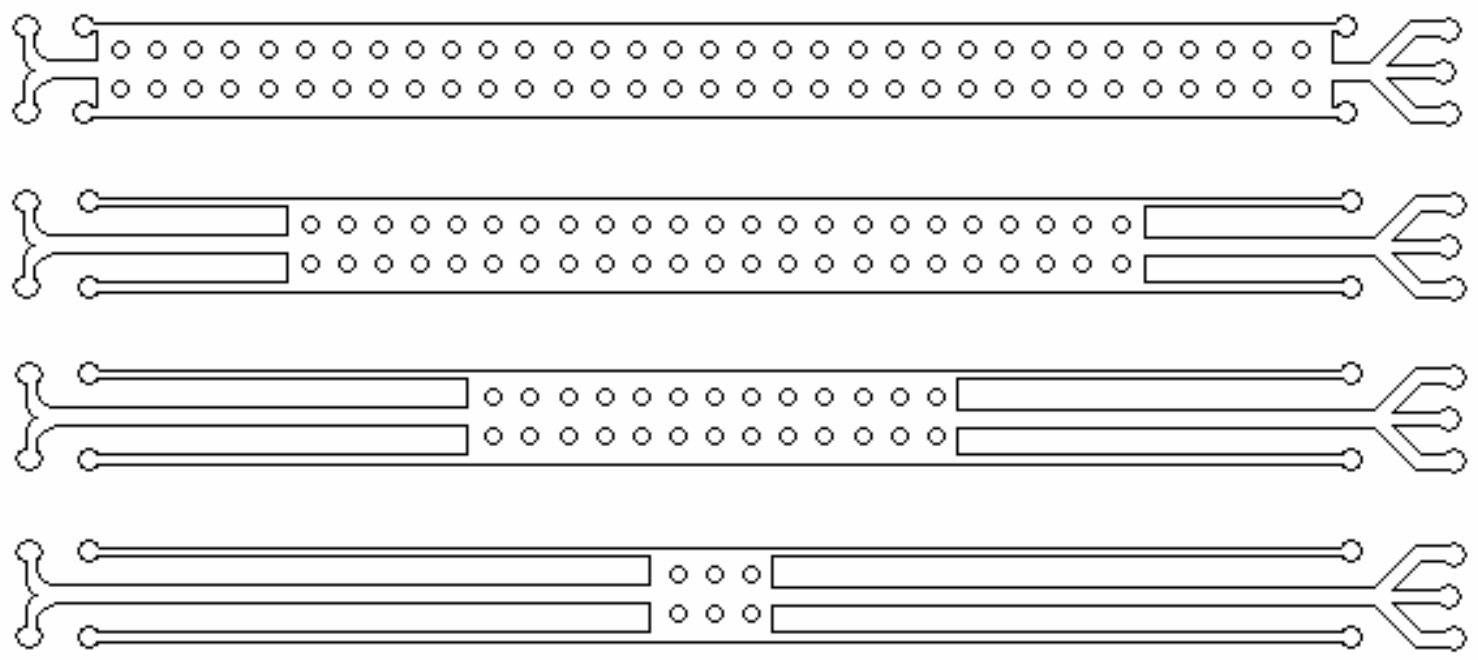

Figure 15. The four chip designs. The designs vary only in the length of the separation channel, which is compensated by the inlet/outlet channel lengths to maintain a constant inlet/outlet interface topography. 
The parallel rows of posts were introduced to prevent the glass from collapsing during the final step of manufacturing, in which the glass is brought to its melting point to join the top and bottom wafers. The posts also serve to add structural support to the gel electrodes which will eventually populate the post region. Figure 16 demonstrates the effect of absent posts.

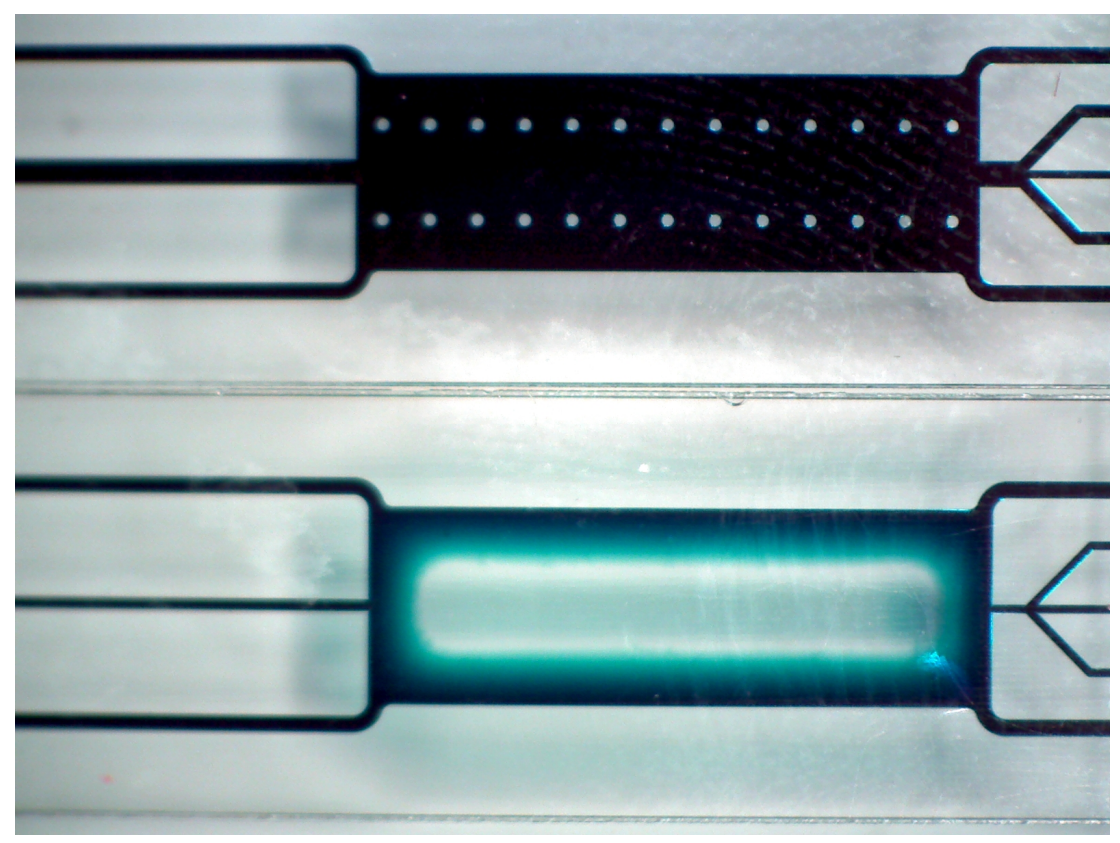

Figure 16. A comparison of chips constructed with, and without posts. In this photograph the chips have been filled with a blue dye to illustrate the reduction in channel depth. The chips without posts have completely collapsed in the center, blocking central fluid flow. They are worthless.

The three branches of the sample channel outlet are intended to isolate each of the three ITP zones: LE, sample, TE. In this version of the chip all three outlet channels have the same width, although in future these widths may be adjusted to divert more of the output into a particular channel. 
Despite the high conductivity of the outside streams, the narrowness of the inlet and outlet channels serve to present a significant electrical resistance, even greater than that of the extremely wide separation channel. For this reason, the applied voltage on the shorter chips is necessarily greater, even beyond what would be necessary to compensate for the reduced residence time.

The longest chip (33mm) was rarely used in development because the edges of the separation channel were not visible under the microscope due to the constraints of the chip mounting.

\subsubsection{Manufacture}

All of the chips used here were manufactured on-site from 500um-thick borosilicate glass wafers. All of the etching and lithography is performed on one side of a single wafer which has been pre-drilled with inlet and outlet holes (drilled off-site). A blank wafer is bonded to the drilled and etched wafer at the end to seal the chips.

\subsection{Gel electrodes}

The polyacrylamide gel electrodes serve two purposes. The first purpose is to separate the sample streams from the high-conductivity LE and TE streams. Balancing the flow of these four streams to achieve a sample channel of uniform width is a monstrous effort, which could possibly require modifications for every single sample 
processed, based on changes in sample viscosity and flowrate. The second purpose is to facilitate the easy transfer of the relative ions from the high-conductivity streams to the sample streams, which would be otherwise impossible with non-porous barriers.

\subsubsection{Design}

The gel electrode should be as narrow as possible while still ensuring structural stability. In the event of a pressure imbalance across the gel, a narrow gel will rupture, causing an unintentional mixing of the high-conductivity and sample streams. The intended spacing between the gels is 500um. With a channel height of $4 \mathrm{~mm}$, the spacing in the image below is 700um.

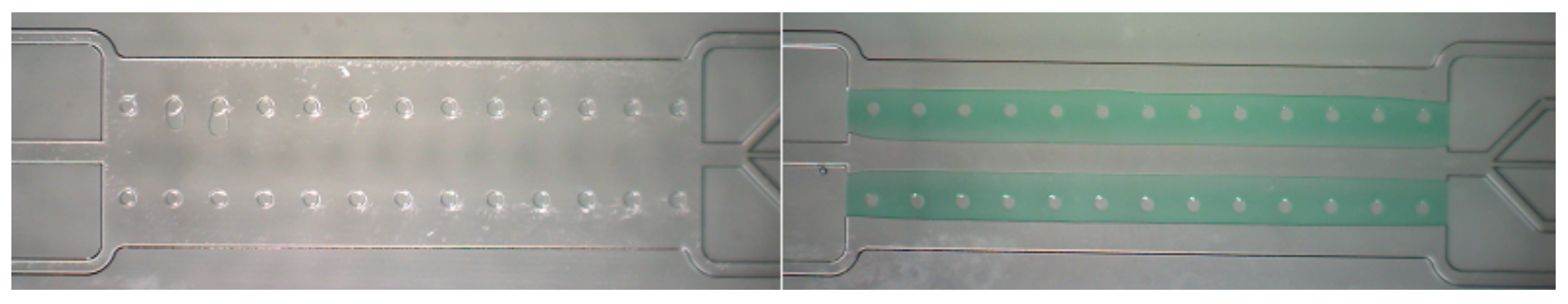

Figure 17. The chip on the left is pre-exposure. The right shows an image of actual gels formed within the chip, taken when the gel processing was at its most sophisticated (in terms of final gel quality and repeatability of results). These are the best gels we can reliably produce.

The gels themselves are not conductive. After the electrodes are set within the chip, the outer channels of the chip are flushed overnight with high conductivity LE and TE, which diffuse into the gel, greatly increasing the gel's conductivity without introducing additional electrolyte species to the system. If the gel loading is not fully completed before being used for ITP, the gels will quickly deplete themselves of the 
diffused ions and return to their original non-conductive state. This overnight loading procedure is one of the hurdles that must be overcome if the transverse ITP chip is to live up to its advertised efficiency and ease of use.

\subsubsection{Manufacture}

Acrylamide gel does not naturally bond to the glass. While it will create a waterand air-tight seal with the glass, the gels are not fixed in place, and thus rupture easily. To combat this nuisance, we employ a technique ${ }^{11,12}$ borrowed from Amy Herr of Sandia National Laboratory. First, the chip is filled with $1 \mathrm{M} \mathrm{NaOH}$ to guarantee that the interior glass surface is clean. After 10 minutes the $\mathrm{NaOH}$ is removed, and the chip is rinsed with DI H2O. The chip is then filled with a 5:3:2 mixture of DI H2O, glacial acetic acid, and 3-(trimethoxysilyl)propyl methacrylate, shorthanded here as "methacrylate." After an incubation of 30 minutes, the methacrylate is removed, and the chip is rinsed with DI $\mathrm{H} 2 \mathrm{O}$ and then with $30 \%$ acetic acid. Figure 18 and Figure 19 illustrate the reasoning behind the methacrylate treatment. The silicon bonds covalently to the interior surface of the borosilicate glass, while the methacrylate group becomes the end of a polyacrylamide chain. The chip is now ready to receive acrylamide. 


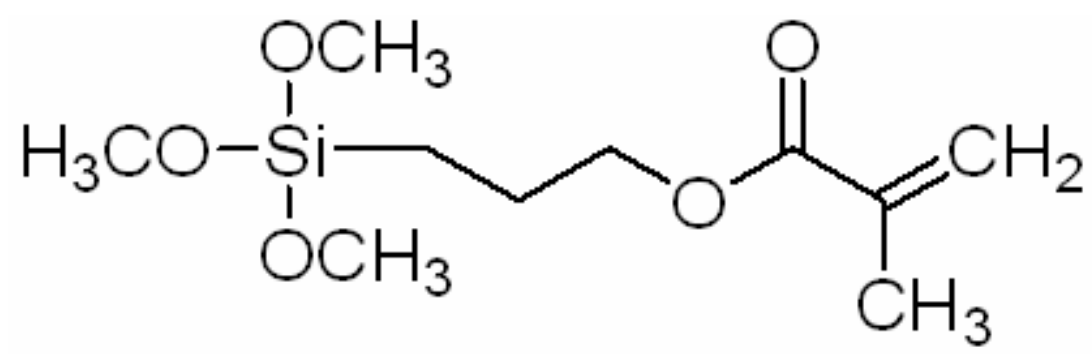

Figure 18. A 3-(tri-methoxysylil)propyl methacrylate molecule. Note the Silicon atom at one end, which bonds with the surface of the glass chip to become part of the crystal lattice, and the acrylamide at the other end which bonds with the surface of the gel as part of the gel matrix.

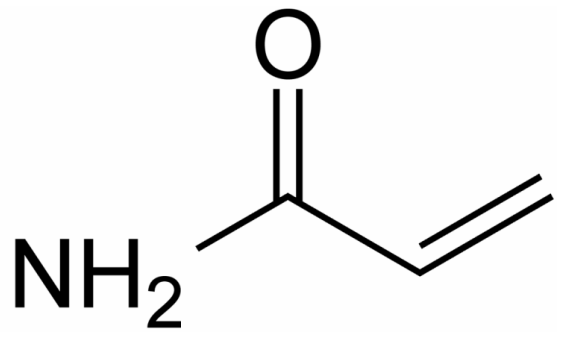

Figure 19. An acrylamide molecule. This is the monomer which contributes the bulk of the mass of the gel, as well as bonding with the free end of the 3-(tri-methoxysylil)propyl methacrylate. A 3-(tri-methoxysylii)propyl methacrylate molecule.

To avoid the hassle of working with dry acrylamide (a potent neurotoxin), we use pre-mixed acrylamide solutions manufactured by Sigma-Aldrich. We began with $30 \%$ acrylamide wbv, with a 37.5:1 monomer: cross-linker ratio. These gels were not as rigid as desired, leading us to switch to a 19:1 ratio, while maintaining the same density of acrylamide. This $30 \%$ acrylamide solution is then mixed 9:1 with a 2\% VA-086 photoinitiator solution, for a final photo-initiator concentration of $0.2 \%$. In the presence of UVlight, the VA-086 splits into free radicals, which catalyzes the polymerization and cross linking of the acrylamide. 
Our UV source is a $350 \mathrm{~W}$ mercury short-arc lamp, with an output power at the exposure plane of $13 \mathrm{~mW} / \mathrm{cm}^{2}$. At this intensity, exposures were completed in 100 seconds, compared to initial exposure lengths of as long as 15 minutes when the bulb output was much lower. We found that all of the inconsistencies in gel exposure which resulted in unusable chips (bridges across electrodes, pinching of high-conductivity streams, inlet/outlet blocking) were the result of unnecessarily long exposures. Partiallypolymerized gel was migrating out of the exposure region, then later polymerizing completely. The switch from eight-minute low-power to two-minute high-power exposures increased the yield of successfully exposed chips from near $20 \%$ to greater than $90 \%$. Previous efforts to reduce the exposure time include increasing the photoinitiator concentration, until we observed that excess photo-initiator resulted directly in unstable gels. After complete exposures and the removal of excess acrylamide, the gels would bleed into the sample channel, then re-polymerize. This effectively destroyed the horizontal nature of the interior gel walls, making them worthless. All efforts to thoroughly consume the photo-initiator in the initial exposure were unsuccessful.

Figure 20 shows the emission spectrum of the mercury lamp at the exposure plane. A quick comparison to Figure 21, the emission spectrum provided by the manufacturer (Advanced Radiation Corporation) reveals that the majority of the UV-light emitted by the lamp never reaches the exposure plane. The attenuation is due to the large lens used to focus the lamp's output, which is made of glass. Quartz lenses which are transparent to UV wavelengths are available, but are also prohibitively expensive. 
Alternatives to the mercury lamp source are being investigated, including UV-emitting LEDs.

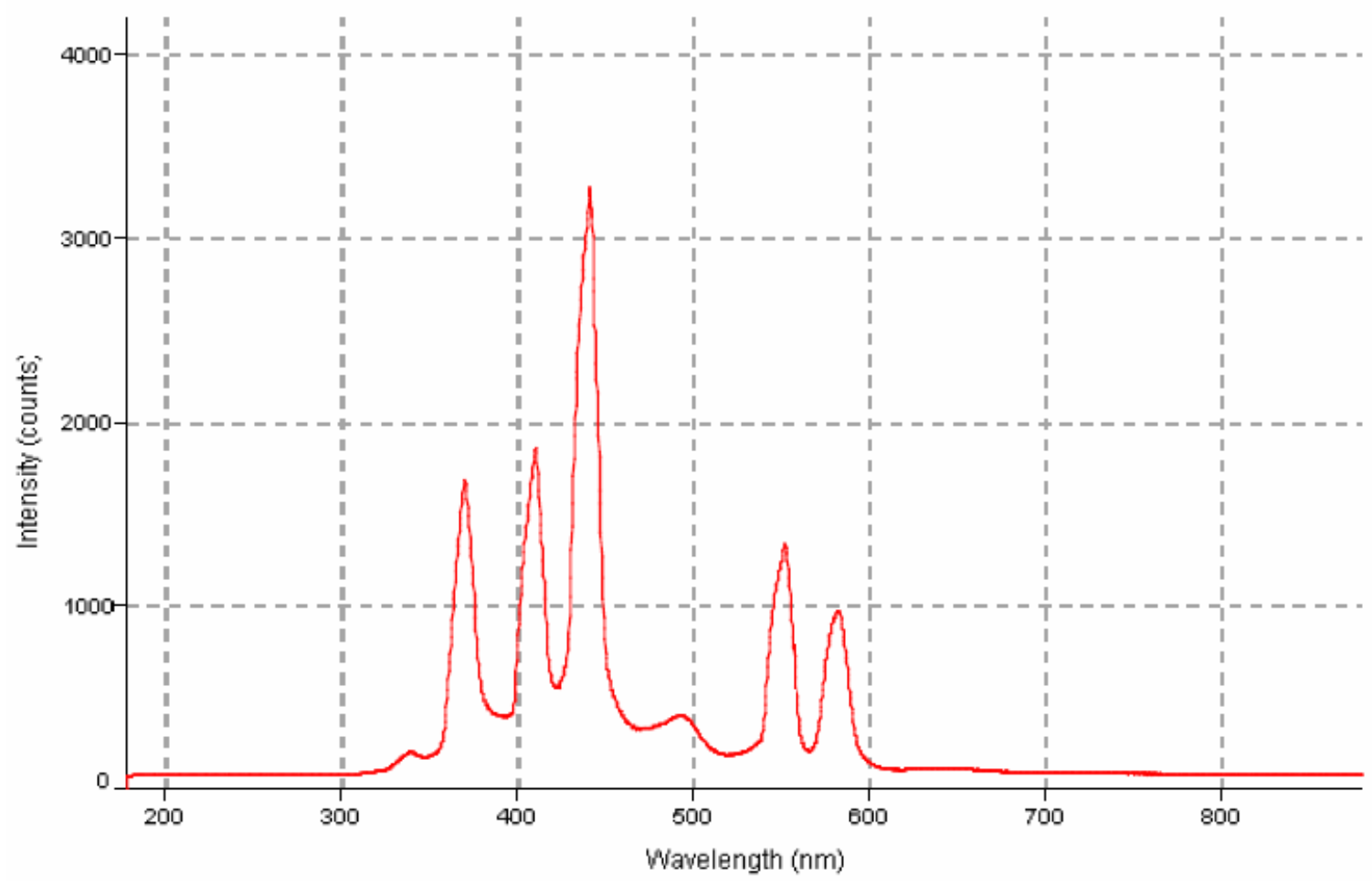

Figure 20. Emission spectrum of the $350 \mathrm{~W}$ Mercury short-arc lamp used to expose the acrylamide gel in the chips. 


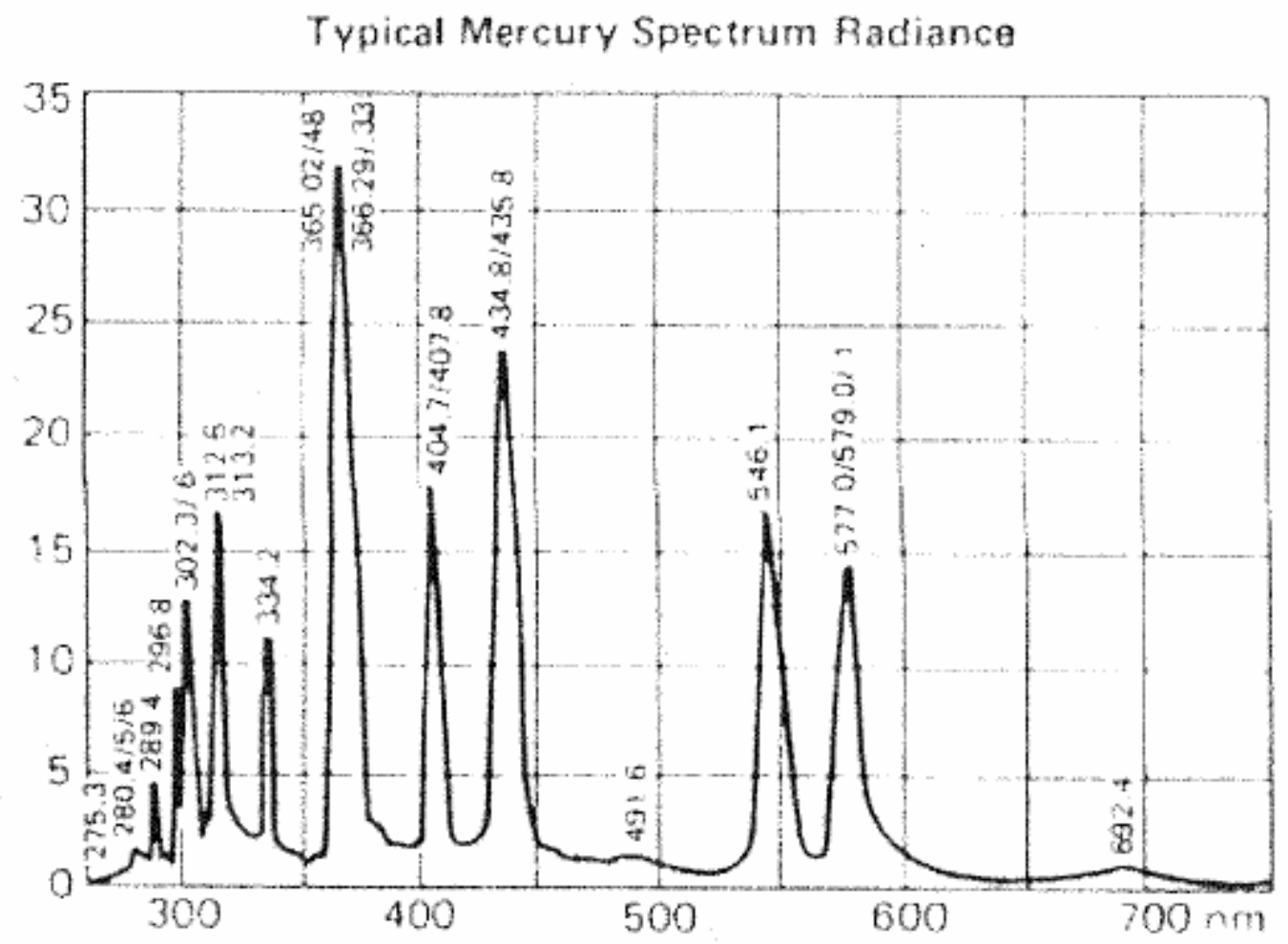

Figure 21. Typical mercury emission spectrum ${ }^{13}$. Note the high population of UV-band peaks conspicuously absent from the lamp output (Figure 21). This image is a scan of printed materials provided by the lamp manufacturer.

Initial gel dimensions were dictated using a mylar mask, which was positioned by hand over the acrylamide-filled chip, and secured with electrical tape. This method was prone to error and inconsistency, leading to the introduction of chrome-on-glass masks. These masks are cut on-site to the same dimensions as the borosilicate chips, which are then placed into an alignment jig prior to exposure. 
The electrodes are exposed individually, with excess acrylamide being removed and replaced between successive exposures. All attempts at single-exposure methods ended poorly, with acrylamide bridges forming between the electrodes. It is likely that an even shorter exposure time could make single-exposure methods viable, but the savings in time would be minimal, and would provide little improvement in gel quality, if any.

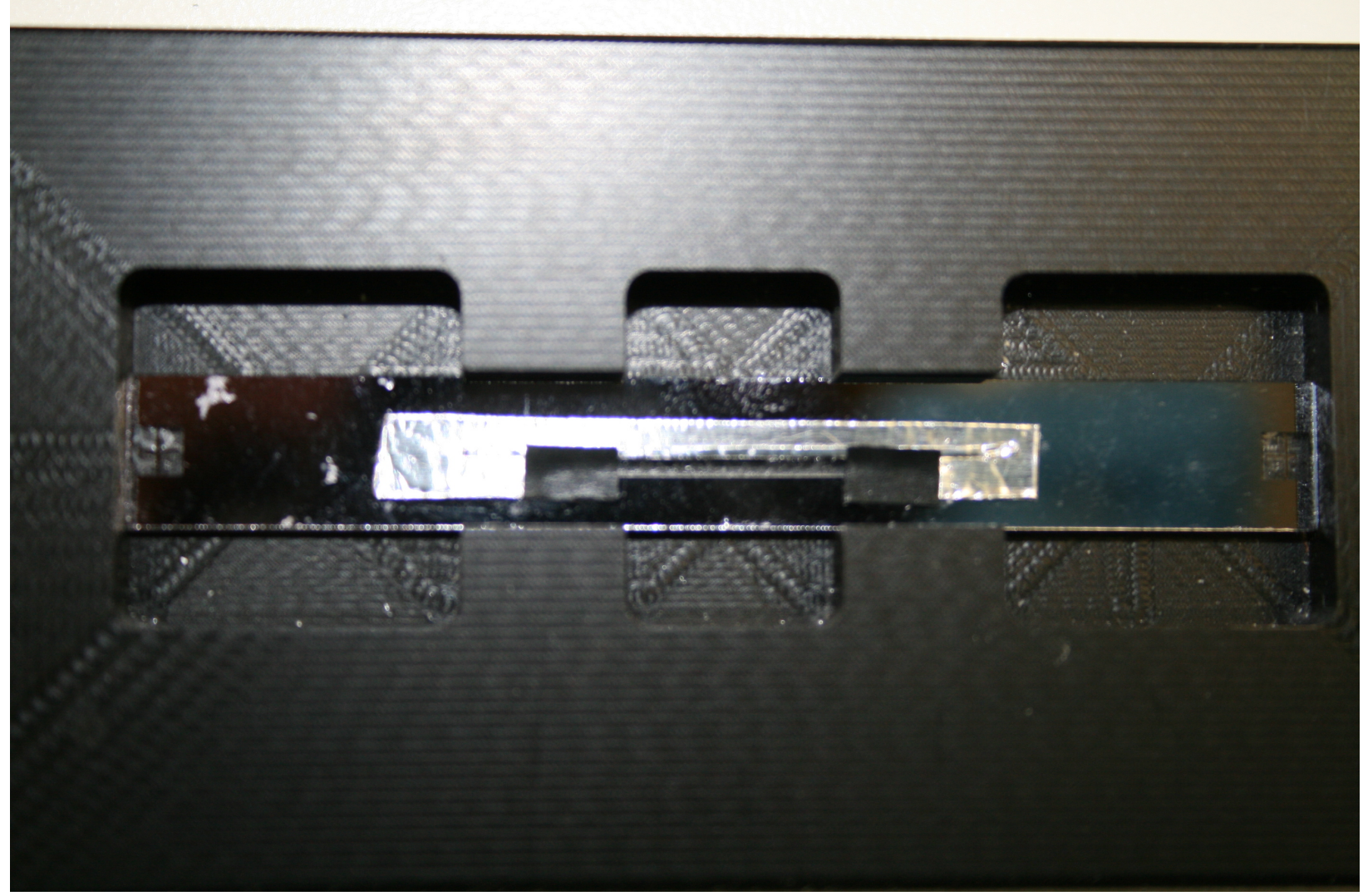

Figure 22. Exposure jig with a chip and mask in place, ready for exposure. Electrical tape has been used to modify the exposure region at the last minute.

\subsection{Paraphernalia}

This section addresses all of the microfluidics accessories used in the actual experiments. Most of this equipment is non-standard and was developed at LLNL 
specifically for use in the microfluidics research there. The construction of the finished products will differ significantly from what is employed here, which was designed for easy modification during the development process.

\subsubsection{Salt Bridges/Nafion Tubes}

The salt bridge is a device manufactured on-site to address the issue of electrolysis. The salt bridge contains two Nafion tubes suspended in an electrolyte reservoir. The Nafion tubes are ion-permeable, such that when a platinum wire is inserted into the reservoir, an electric field may be carried by the fluid within the Nafion tube without excessive bubbles forming within the electrolyte stream.

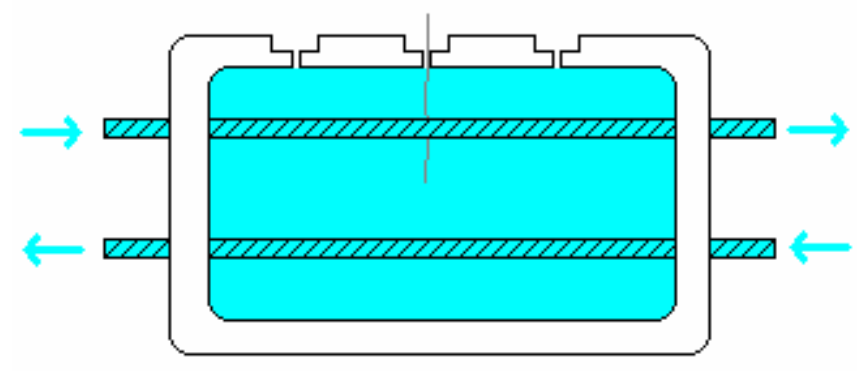

Figure 23. The salt bridge isolates the on-chip high-conductivity streams from the bubbles produced by electrolysis at the platinum electrode.

\subsubsection{Breadboard}

The microfluidics breadboard is a development platform designed specifically for use at LLNL. It features an array of threaded holes which we use both for mounting the chips and delivering the reagents. LE and TE inlet and outlet tubing (0.03"' ID) mount on the breadboard via hollow-center threaded fittings. The breadboard itself rests in a 
complementary depression on a microscope stage, with the inlet and outlet tubing extending away from the board on the side which is not visible to the scope camera.

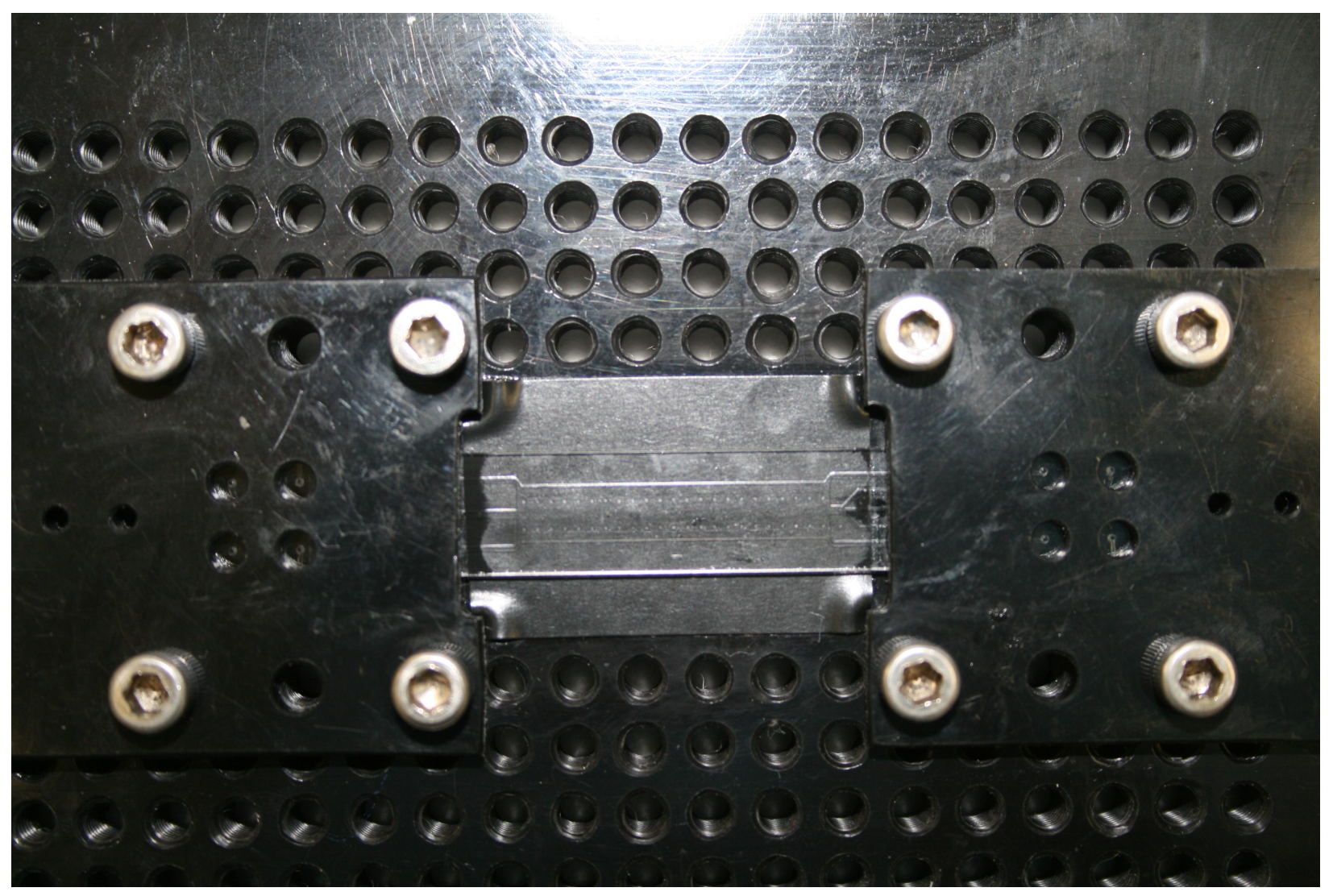

Figure 24. The breadboard used to facilitate the interface between on-chip channels and off-chip reservoirs. The chip has been mounted and is ready to receive the ITP reagents.

\subsection{Buffers/Electrolytes}

The choice of leading/trailing electrolytes changes with every ITP, as well as the $\mathrm{pH}$ of the LE/TE. Buffers are most effective when titrated to their $\mathrm{pKa}^{14}$, which is usually the deciding characteristic in buffer selection. The LE/TE need not always serve as the buffer, as HEPES often does. Tris is an example of a buffer which is neither the leading 
nor trailing electrolyte (but it is often titrated with chloride, which is a leading electrolyte).

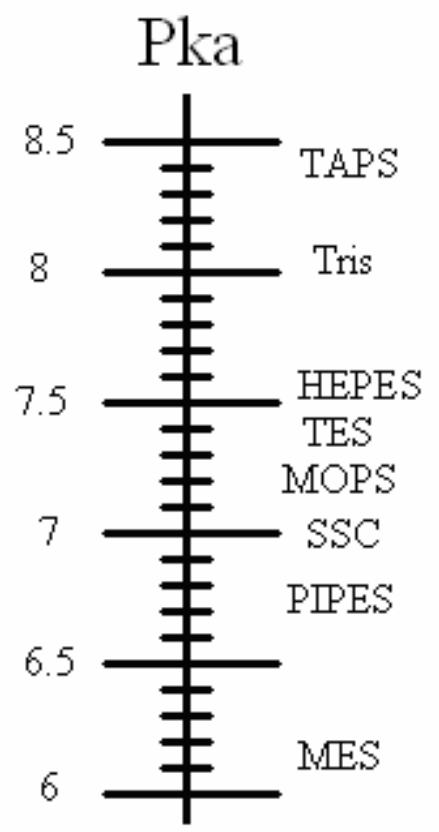

Figure 25. Common buffers and their $\mathrm{pKa}^{15}$. It should be used in conjunction with Figure 1 when selecting buffers for an ITP. 


\section{Results}

All of the images in this chapter are of fluorescein, which has an electrophoretic mobility similar to that of DNA. All images were taken with a FITC filter, which allows incident blue light to pass through to excite the fluorescein, and emitted green light to pass back, which is then seen by the camera. FITC stands for Fluorescein Isothiocyanate, the fluorescent dye for which the filter was originally developed. The concentration of fluorescein at any location is directly proportional to the intensity (brightness) at that location.

\subsection{Capillary ITP}

The following figures demonstrate traditional ITP carried out in a borosilicate glass capillary, manufactured by Wilmad LabGlass. The dimensions of the rectangular capillary shown here are 50um x 500um, with a length of $10 \mathrm{~cm}$. In Figure 26, we see that the unfocused band collects into a brighter band after the electric field is established, as expected. Figure 27 shows that once the band is fully collected, its dimensions are relatively constant, and it moves at a constant speed. The capillary is long enough in this example that the electric field may be considered constant over the timescales in which these pictures were taken. In each figure, one second elapses between successive frames. 


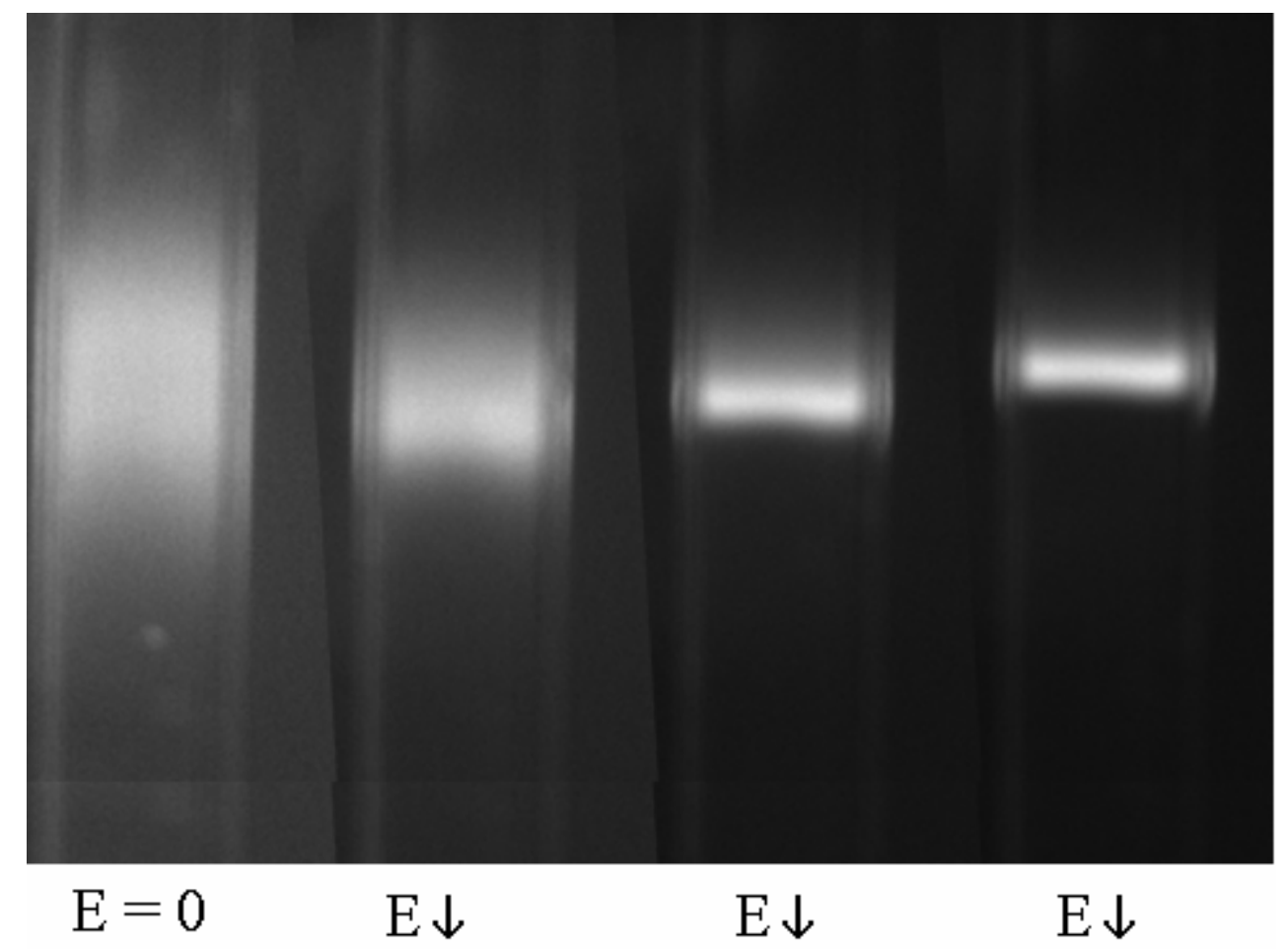

Figure 26. ITP in action. The initial unfocused sample of the leftmost frame is focused into the confined band seen in the rightmost frame. 


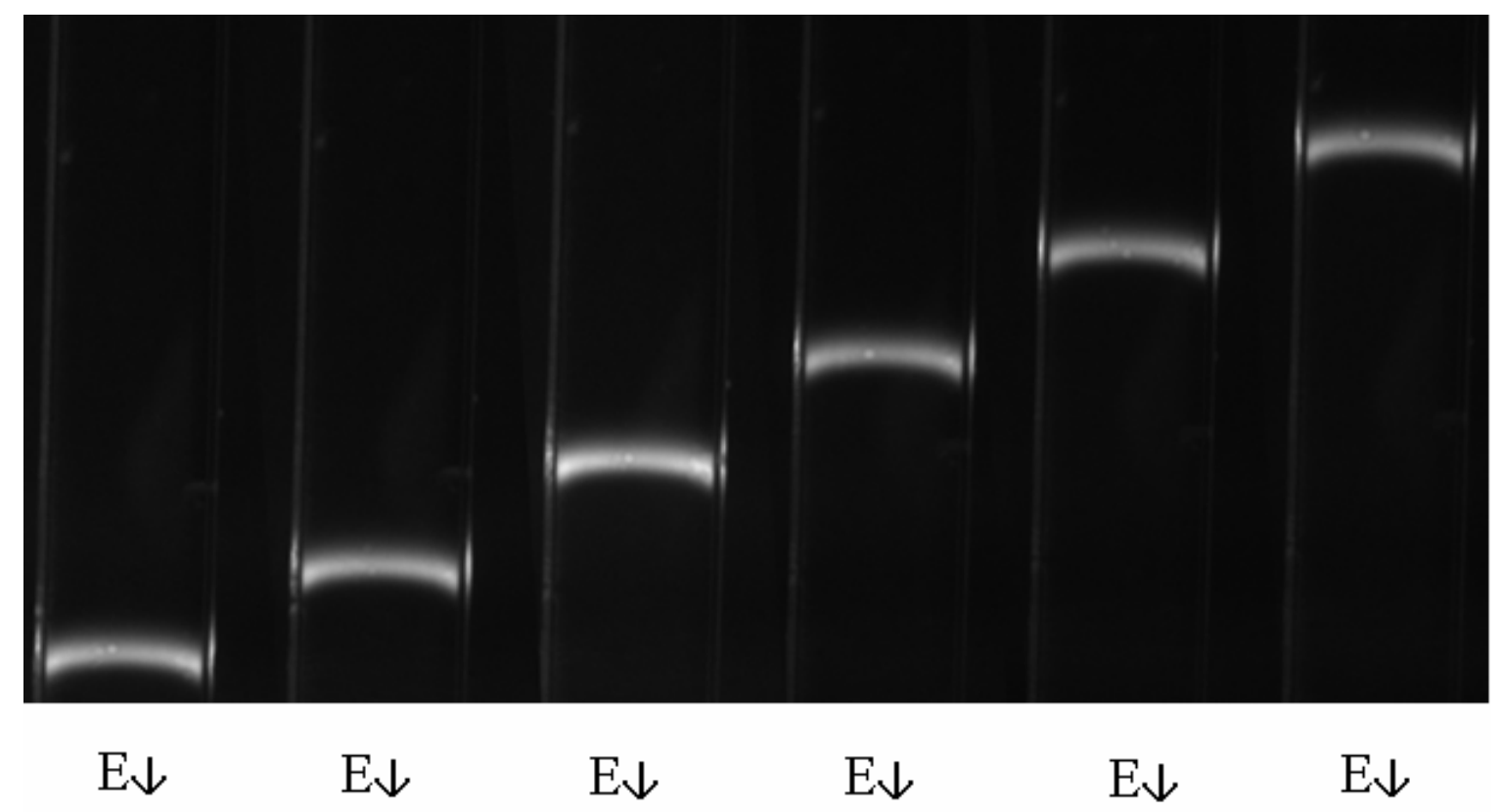

Figure 27. Maintaining a focused band. Note that the band is more diffuse on the LE side, as predicted by Figure 11.

\subsection{Surrogate TE}

To test the viability of our design we first tried the classic ITP setup, with the dye in the TE. The LE was $100 \mathrm{mM}$ Tris $/ \mathrm{HCl} \mathrm{pH}=8.0$, and the TE was $10 \mathrm{mM}$ Hepes $/ \mathrm{NaOH}$ $\mathrm{pH}=7.5+20 \mathrm{nM}$ fluorescein. The sample flowrate in these pictures is $100 \mathrm{uL} / \mathrm{min}$. 


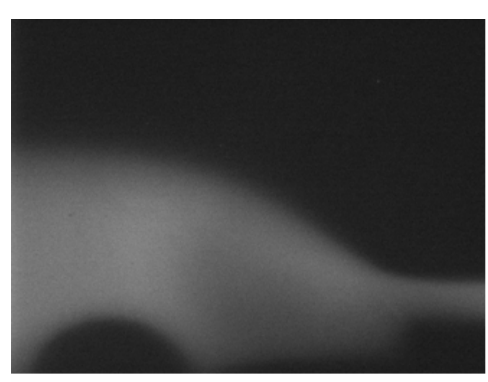

$200 \mathrm{~V}$

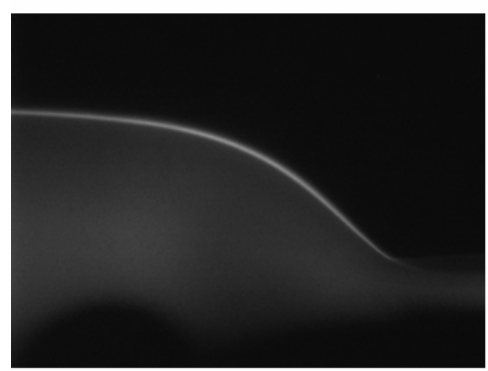

$500 \mathrm{~V}$

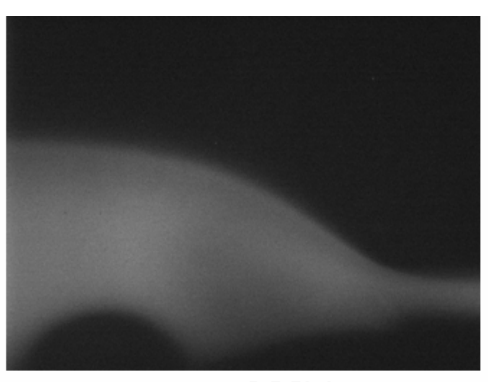

$300 \mathrm{~V}$

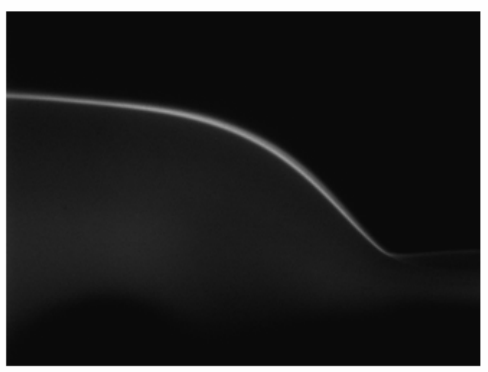

$600 \mathrm{~V}$

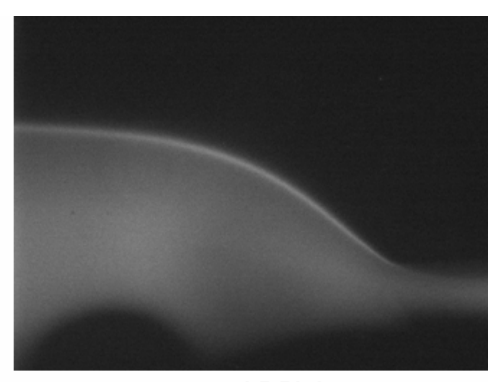

$400 \mathrm{~V}$

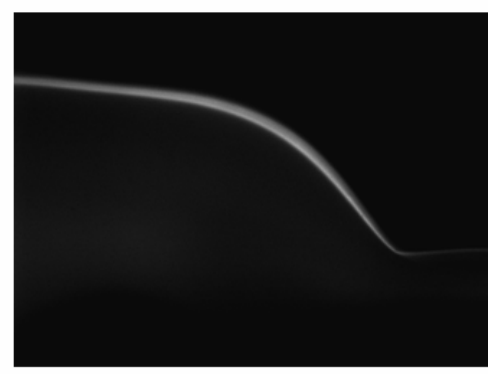

$700 \mathrm{~V}$

Figure 28. Outlet of a transverse ITP chip. The downward slope is due to the failure of the purely-horizontal fluid flow approximation where the channel suddenly constricts. Note the less confined state of the band on the LE side of the $700 \mathrm{~V}$ frame. The $200 \mathrm{~V}$ frame is nearly indistinguishable from the OV case (not shown).

The black bump seen at the bottom of each frame is not an effect of the ITP, but rather an effect of the imperfect gel-forming techniques used at the time these were taken. All of these images are taken at the outlet of a $23 \mathrm{~mm}$ chip, where the band is most focused. Note that the sudden downward turn is not an electrical effect, but is due merely to the constriction of the channel at the outlet. Newer versions of the channel do not feature this constriction, as it results in an unexpected loss of band fidelity before the outlet stream splits. From a qualitative standpoint, it is evident from the photographs that the band quality increases with increased voltage. At $200 \mathrm{~V}$ it is apparent that no focusing has occurred, while at $700 \mathrm{~V}$ one could justly say that there is almost no focusing left to be done. For this particular setup $700 \mathrm{~V}$ was the practical limit of the applied voltage, as 
increasing it any further caused the band to pass into the gel, from which it is not recoverable. The 700V image is reproduced in Figure 29.

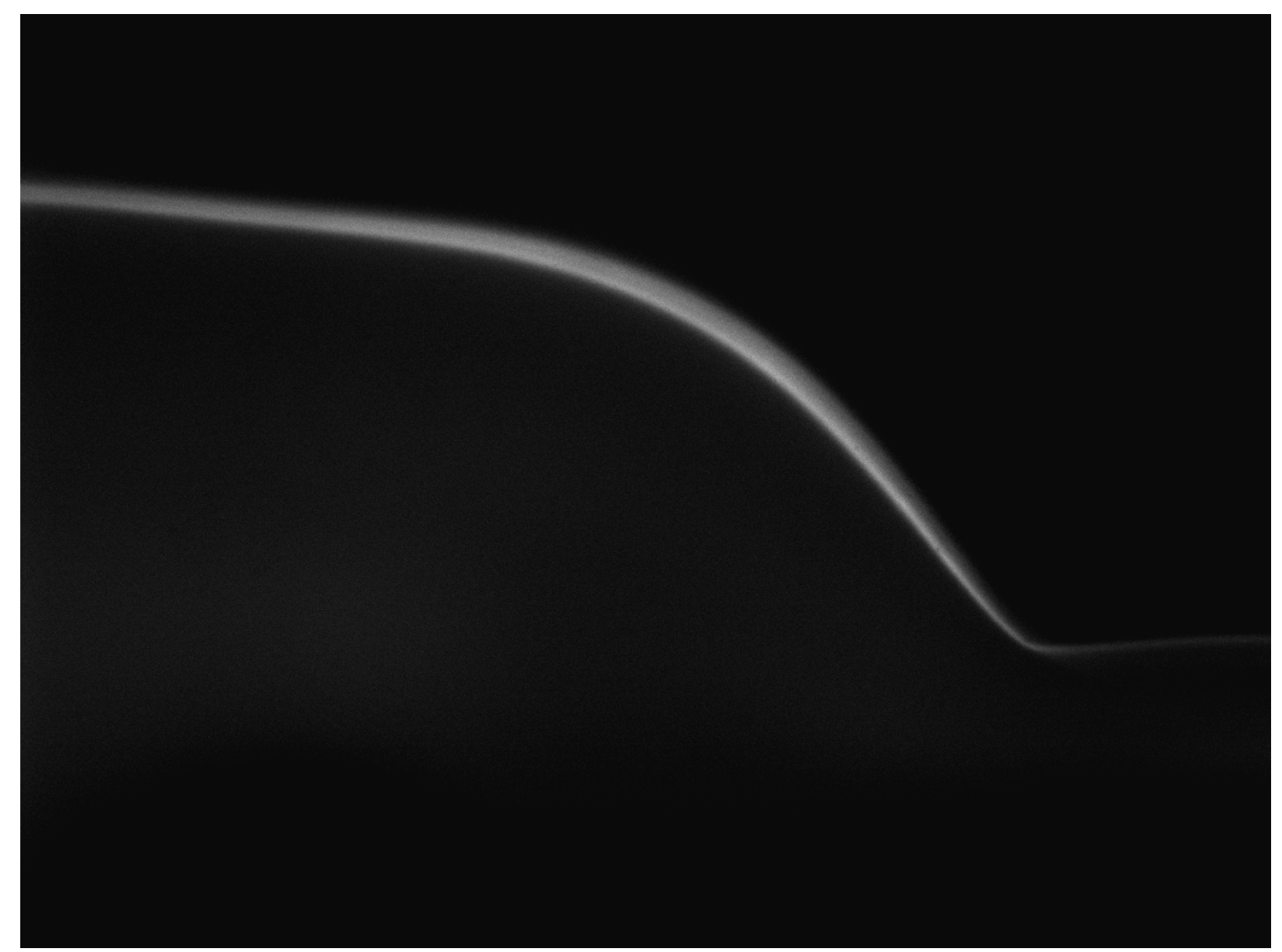

Figure 29. An enlarged view of the $700 \mathrm{~V}$ frame offigure 28. The TE region is not entirely free of fluorescein, although that is more closely achieved at slower speeds.

With Figure 29 enlarged thusly, we see that the area beneath the band is not entirely black. What little fluorescein remains unfocused is not worth reclaiming, although it is possible. Figure 30 shows the same device, operating at $700 \mathrm{~V}$ at $75 \mathrm{uL} / \mathrm{min}$. 
The band is noticeably narrower in this image, and it appears that there is no fluorescein left beneath the band, but these minor gains are hardly worth the $33 \%$ increase in processing time.

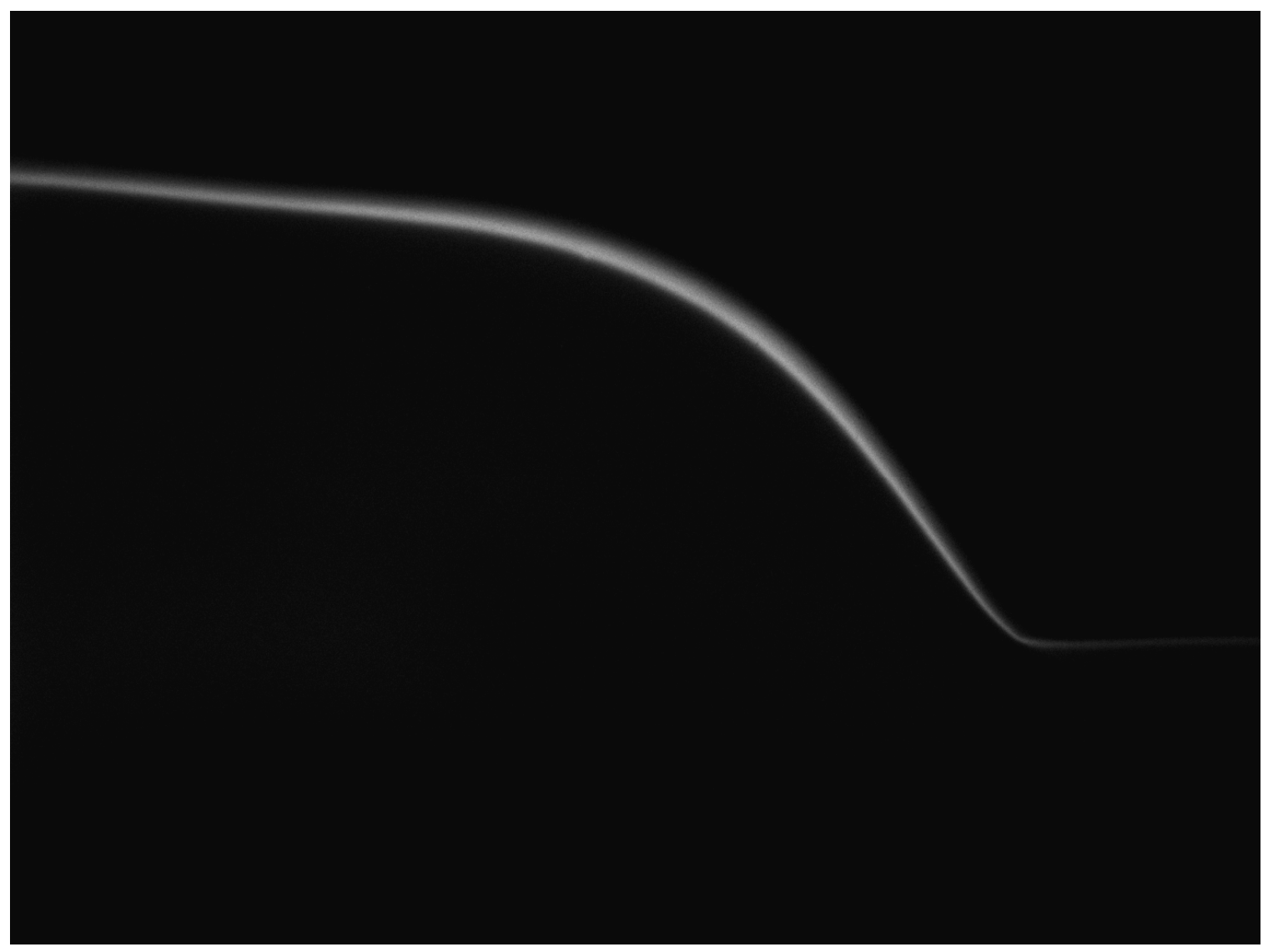

Figure 30 . The same device and applied voltage as Figure 28 , but run at $75 \%$ speed. Here the lagging fluorescein of Figure $\mathbf{2 8}$ has joined the band, which is noticeably more confined.

\subsection{Surrogate LE}

With the proper operation of our chip verified (minus gel imperfections), we move on toward actual samples. In addition to blood, our device is intended also to accepted saline sinus-rinse as an input. This saline rinse is mostly $\mathrm{NaCl}$, and since $\mathrm{Cl}-$ is our leading electrolyte, we simulate the saline rinse by doping the LE with fluorescein, 
rather than the TE as was seen previously. The effectiveness of this method is expressed entirely by Figure 31.

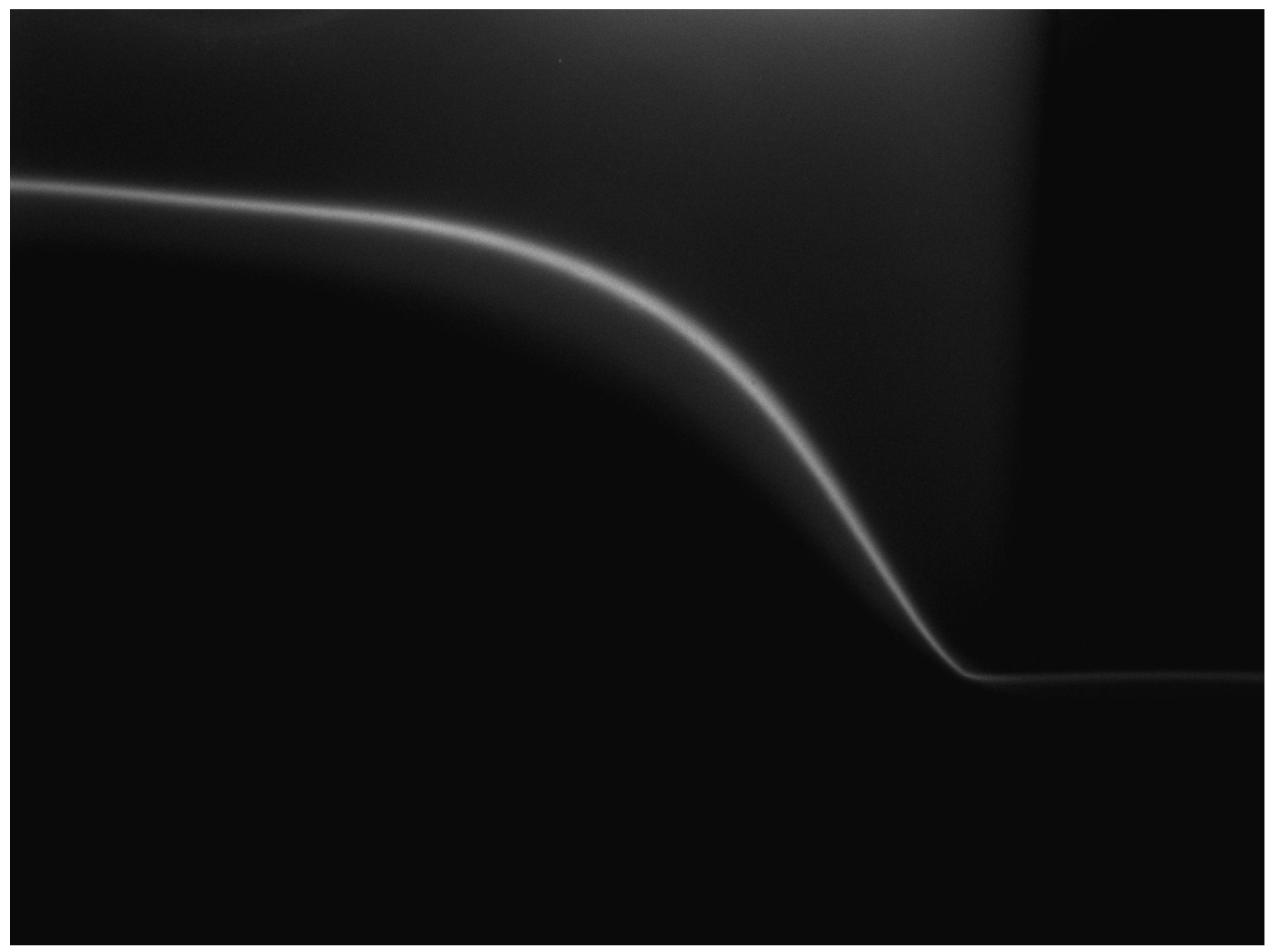

Figure 31 . We achieve the focused band here with considerably less voltage (200V) than in Figure 28 (700V). However, the band is not of comparable quality to that of Figure 28 because a considerable amount of sample has been lost to the gel via electrophoresis.

The focusing seen in Figure 31 was achieved at $200 \mathrm{~V}$ and $100 \mathrm{uL} / \mathrm{min}$. This is significantly less voltage than was required by the previous setup, but it should be noted that the band is not as bright here as it appears to be. The imaging software automatically 
normalizes the brightness to the brightest object in the field of view, such that this band is significantly less bright than its counterpart on the previous page. It is also evident here that significant sample has escaped into the gel, from which it is not recoverable. Worse still, is that the band occupies (in vertical space alone) the original sample region. If this band were to be reclaimed, it would be anything but pure. Any slower or non-charged constituents of the initial sample will be present in the output stream, contrary to the goal of the project. If the sample is to be contained within the LE, the band must pass entirely out of the initial LE region before it is reclaimed.

\subsection{Dual-LE Input}

In this setup there is no dilute TE input. The two sample streams are identical solutions, except that the input adjacent to the TE gel contains $20 \mathrm{nM}$ fluorescein, as illustrated in Figure 32. 


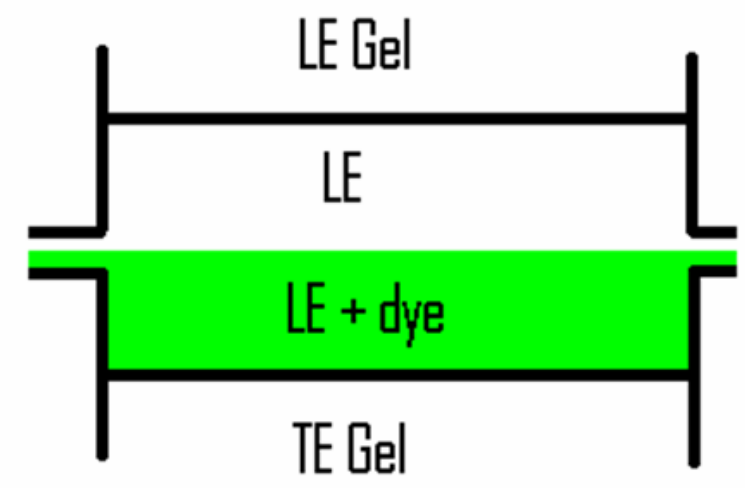

Figure 32. Dual-LE input setup. The top LE band is of a higher concentration than the Le + sample band, in order to ensure a higher electric field in the sample region.

The LE in this case is $10 \mathrm{mM}$ Tris/ $\mathrm{HCl} \mathrm{pH}=8.0$. The effectiveness of this setup is illustrated in Figure 32. Note that the sample flow rate in these photographs is only $25 \mathrm{uL} / \mathrm{min}$ (compared to $100 \mathrm{uL} / \mathrm{min}$ for the traditional LE/TE setup). 


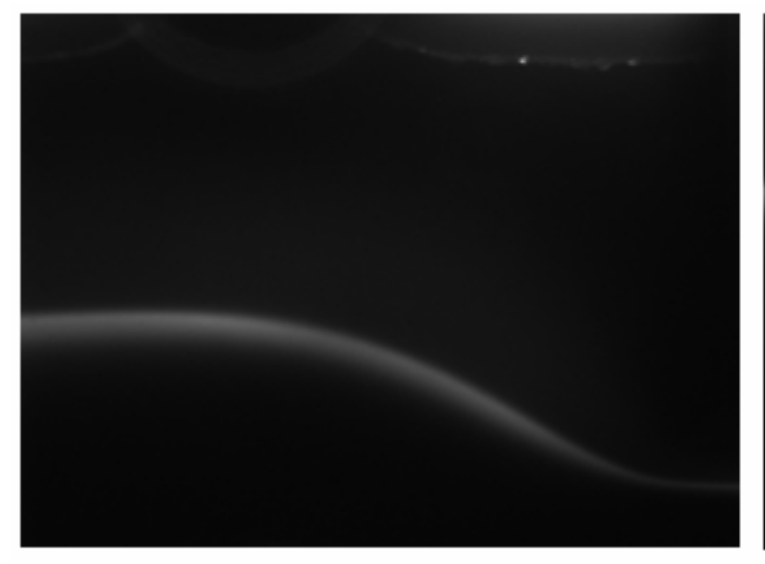

$200 \mathrm{~V}$

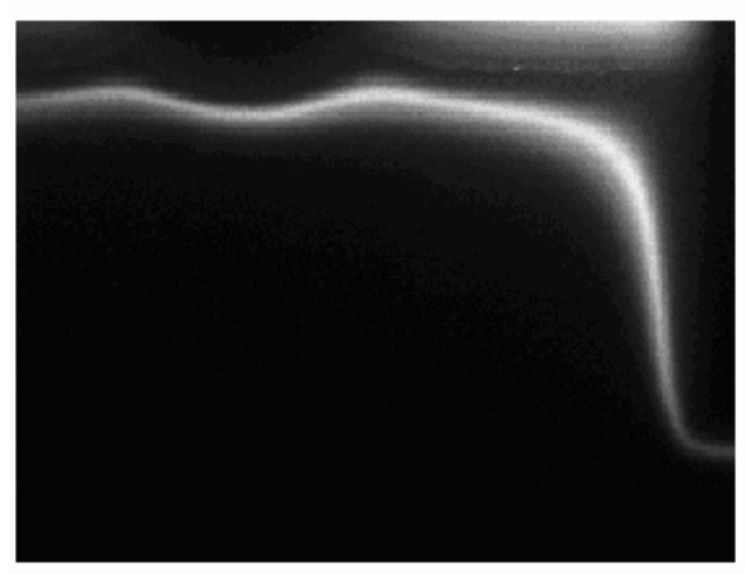

\section{$400 \mathrm{~V}$}

Figure 33. The dual-LE setup under varying voltage. The $400 \mathrm{~V}$ frame shows the focused band bleeding into the gel under an excessive input voltage. The "bump" in the band is due to an aberration in the gel wall.

As expected, the band quality increases with increased voltage, until we see at $400 \mathrm{~V}$ it has just barely begun to dip into the LE gel. The band is actually so near to the gel that the imperfections in the gel are readily seen via their effect on the band position. Despite running only at $25 \mathrm{uL} / \mathrm{min}$, the presence of the band confirms the viability of the dual-LE input setup. 


\subsection{1x NeilMed Input}

Upon the success of the dual-LE input ITP, we move again closer to actual sample conditions. We brewed one packet of the NeilMed sinus rinse saline solution as per package directions, and then diluted it by a factor of ten. This $0.1 \mathrm{x}$ solution was used for both inputs, with the one nearest the TE gel again carrying 20nM fluorescein. The $0.1 \mathrm{x}$ NeilMed solution is roughly six times more conductive than the Tris/ $\mathrm{HCl}$ buffer previously used, which was itself pushing the limits of the low-conductivity requirement for a uniform field, as dictated in Chapter 3. We expected that the $0.1 \mathrm{x}$ NeilMed solution would be too conductive, resulting in an unacceptable sag in the electric field and a definite decrease in band quality, if any band appeared at all. Figure 34 illustrates the expected decrease in electric field quality. 


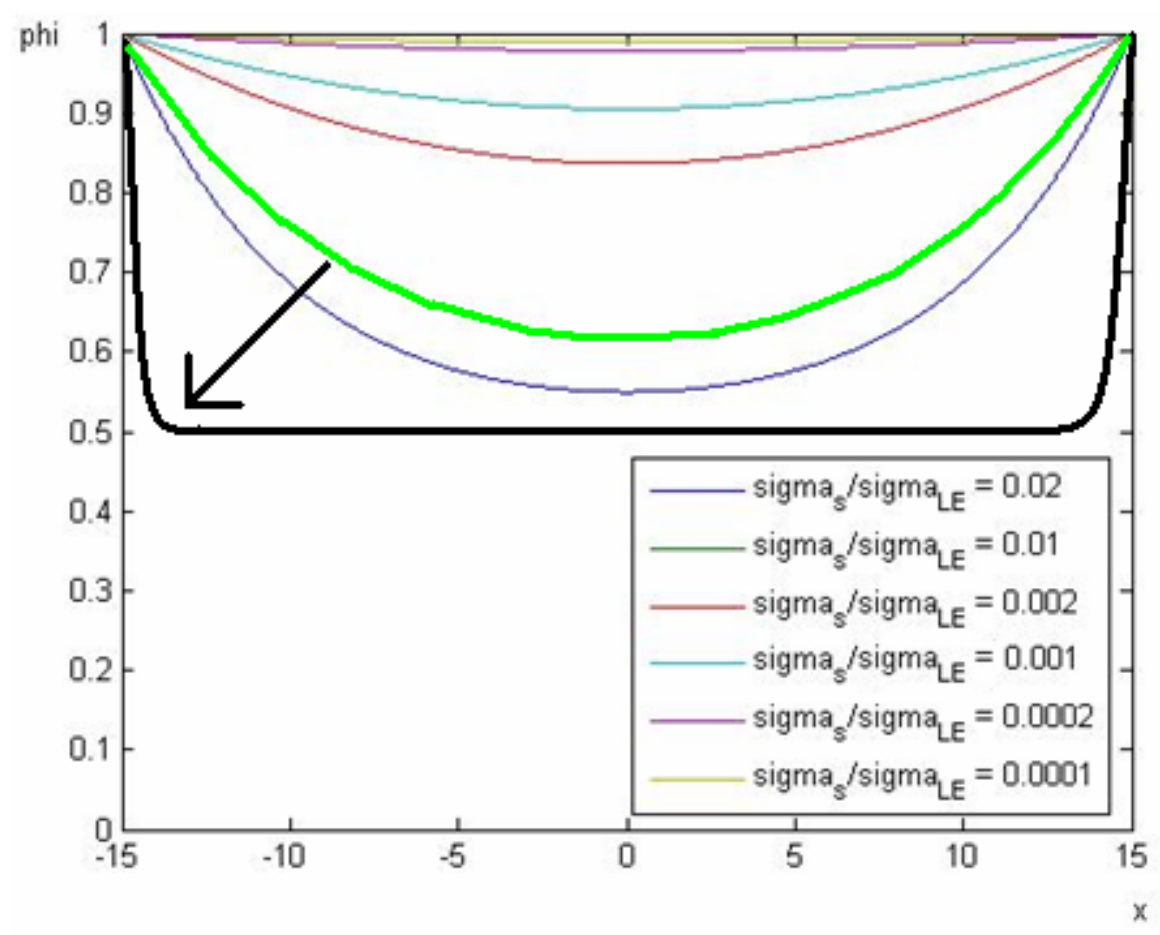

Figure 34. The expected electrical performance of the $0.1 x$ NeilMed input. The black line is only an estimate of the resulting potential, which is beyond the realm of reasonable assumptions used to produce the other traces in the figure.

In Figure 34, the green line is where the dual-Tris/ $\mathrm{HCl}$ input was operating. The black line is the expected characteristic of the dual-0.1x NeilMed setup. Note that this is only the plot for the LE potential. The TE potential (not shown) is a mirror image, flipped across the line phi $=0.5$, such that for poor conductivity ratios, as we are about to see, the valley of one meets the peak of another and there is no measurable potential difference.

Figure 35 shows the changes in band quality over a larger range of voltages than have previously been used. In all frames, the sample flow rate is $50 \mathrm{uL} / \mathrm{min}$. 


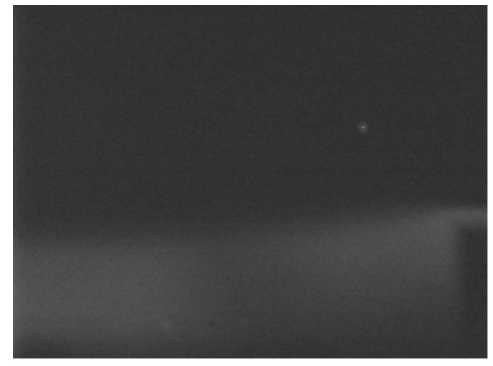

OV

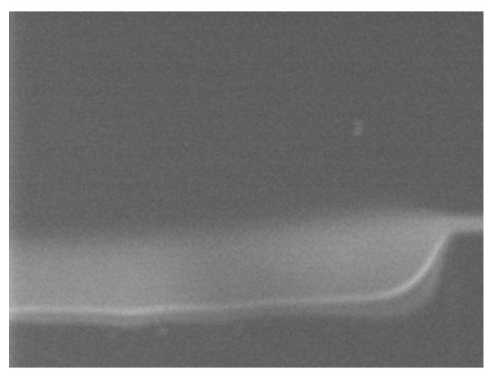

$1200 \mathrm{~V}$

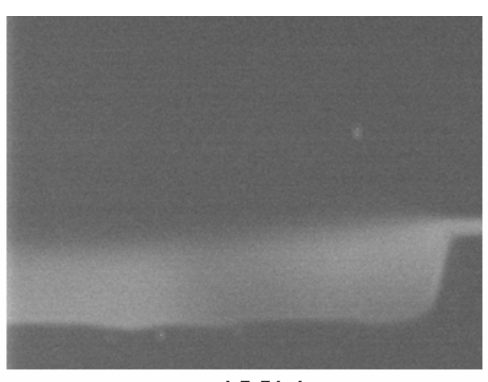

$400 \mathrm{~V}$

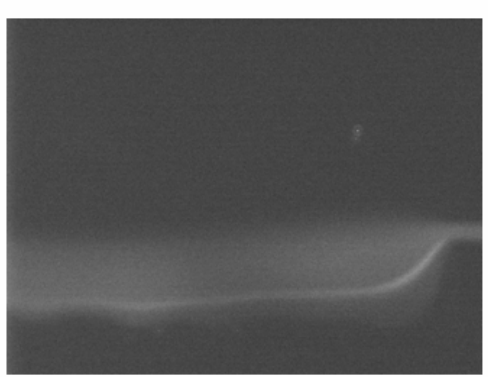

$1600 \mathrm{~V}$

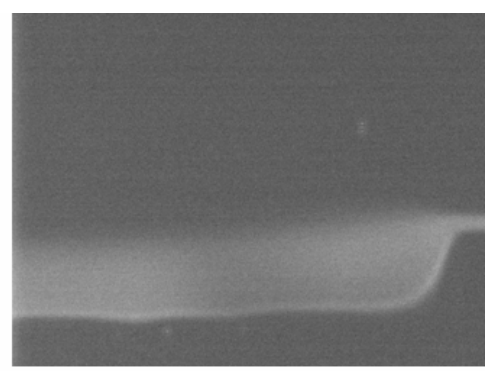

$800 \mathrm{~V}$

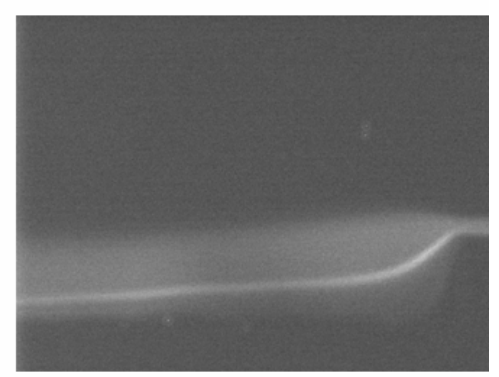

$2000 \mathrm{~V}$

Figure 35. The outlet of the $0.1 \times$ NeilMed trials. Note that under considerably high voltages (200V) and reduced flowrates (50uL/min), the focused band has failed to clear the initial sample region, which would guarantee contamination in the recovery stream.

We expect that the band quality should suffer if the flowrate is increased, as this translates directly to reduced residence time. However, we also expect that a 10x increase in voltage would more than compensate for the decreased residence time. Even at 2000V, the fluorescein band fails to escape the initial sample region. The increase in applied voltage (700V to $2000 \mathrm{~V}$ ) does not compensate for the apparent collapse of the potentials in the high-conductivity streams. Figure 36 is an enlarged reproduction of the $2000 \mathrm{~V}$ image. We see here that the region beneath the band is not even near to being entirely free of fluorescein. Not only is the band not reclaimable in its current state on the basis of 
purity, but to reclaim it would also require the forfeiture of a significant portion of the sample.

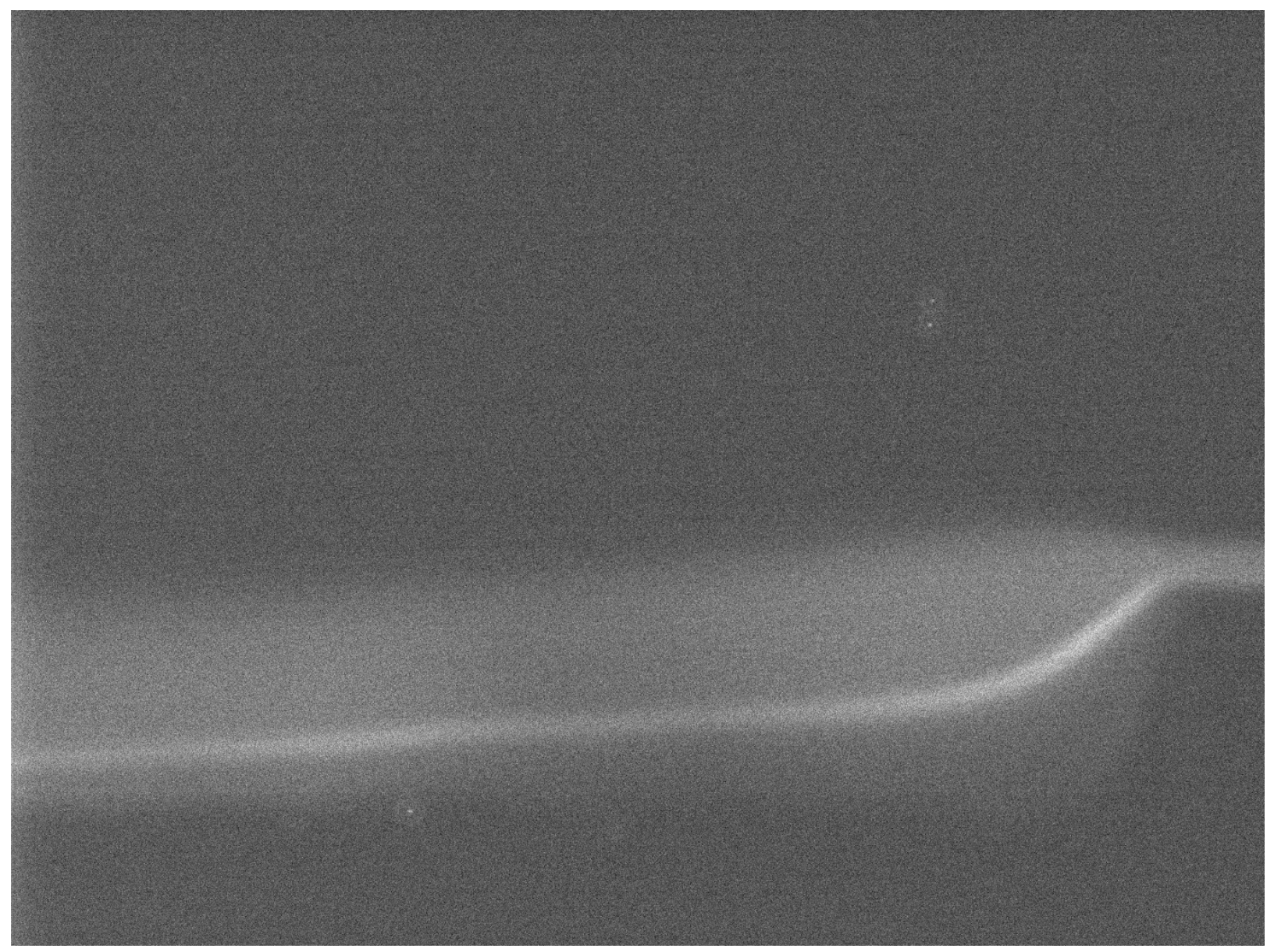

Figure 36. Enlarged view of the $2000 \mathrm{~V}$ frame of Figure 34.

We must resolve the collapsing potential issue before experimentation can move to a $1 \mathrm{x}$ NeilMed solution 


\subsection{Gel Response}

All is not well in paradise. For all of the qualitative results evident from the preceding figures, we have no quantitative data available to test the mathematical models of Chapter 3. The volume/boundaries of the polyacrylamide gels are not fixed, even after they have been set within the chip. Initial gel boundaries can be damaged by the electrolyte loading process, resulting in an uneven gel wall which contributes to both non-horizontal flow and a non-uniform electric field. Application of high voltages only worsens the gel boundary quality, in a seemingly irreversible way. Above all, the origin of the coordinate system used in the model is constantly changing as the TE gel wall encroaches upon the separation region. These effects are shown in Figure 37.

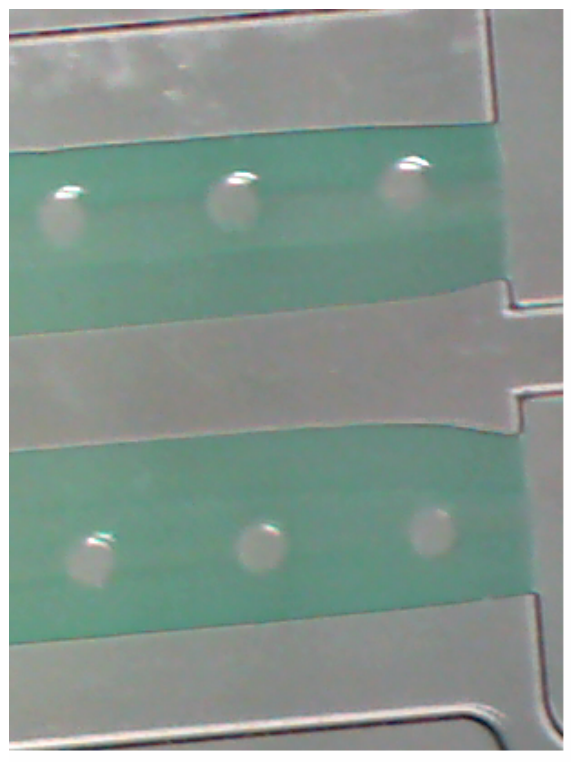

Fresh from the lamp

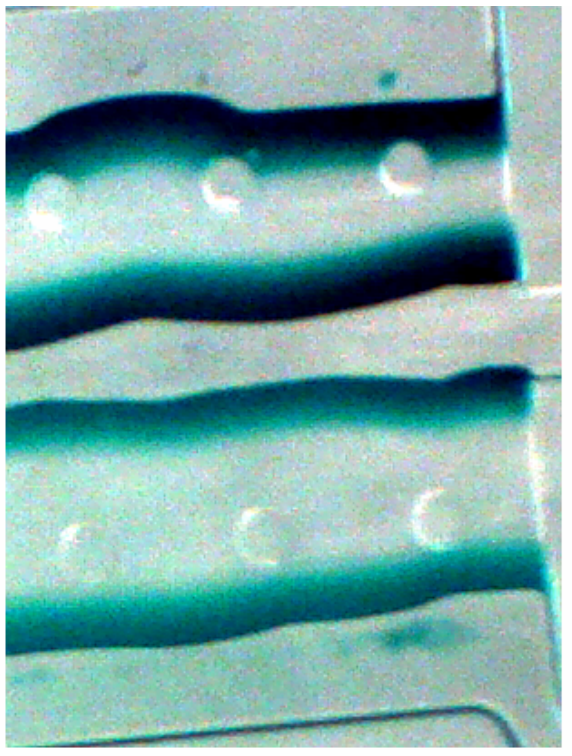

Dvernight Loading

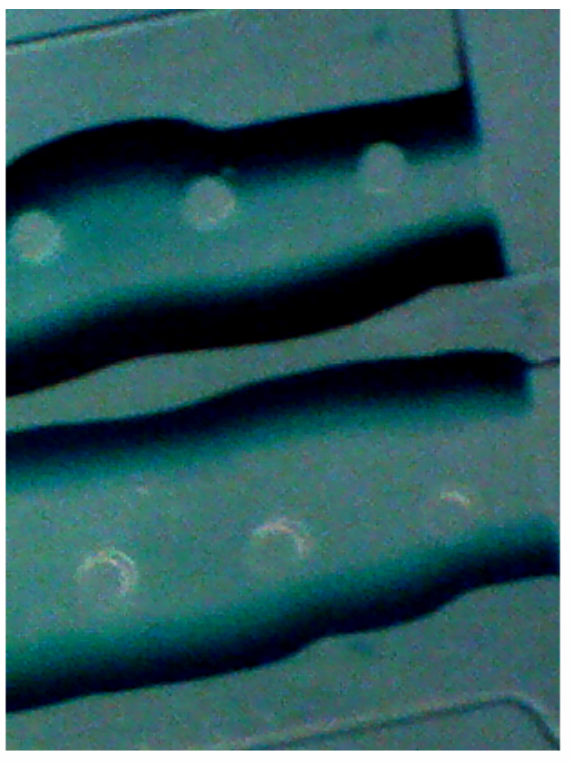

Post-ITP

Figure 37. Degradation of gel quality at various stages of use. 
From Figure 37 it is plainly evident that any attempt at taking quantitative measurements would be futile until such time as the gel boundary issues are reconciled. Suggested fixes include increasing the $\mathrm{x}$-link : monomer ratio, reducing the total acrylamide concentration, and modifying the gel-setting process to remove potentially unpolymerized acrylamide before a final curing step. 


\section{Conclusion}

The (poly)acrylamide gel electrode approach to transverse ITP is a promising method of sample isolation, although it is not yet ready for widespread use. The models developed in Chapter 3 make predictions of the necessary conditions for successful ITP, including gel/chip dimensions, electrolyte concentrations, and applied voltage. Chapter 4 outlines the current manufacturing process of the transverse ITP chip, and it is expected that validation of the models of Chapter 3 will lead to changes in the methods/parameters of Chapter 4. Chapter 5 demonstrates the successful isolation and concentration of surrogate samples in a variety of input configurations, including TE + sample, LE + sample, and dual-LE. The repetition of these victories with biological samples in place of surrogates would demonstrate a completely successful transverse-ITP setup.

\subsection{Future work}

Future work with transverse ITP should focus on resolving the wandering gel-boundary issue, which is at this writing the major roadblock to verifying the models of Chapter 3. Possibilities to explore are:

1) Reducing the concentration of the gel in solution prior to curing.

2) Removing excess/unexposed gel between curing steps.

3) Increasing $x$-link: monomer ratio.

4) X-linker dynamics under high electric fields. 
While it would be nice to maintain all current work with acrylamide gels, it may be worthwhile to try different media if the necessarily high electric fields are responsible for a structural failure within the gel.

The other major barrier to successful ITP is the sagging of the electric field in the LE/TE regions due to high-conductivity samples. Possible fixes for this are:

1) Wider/deeper LE/TE side-channels to reduce effective resistance of those channels.

2) Replacing the long side-channels with two or more shorter channels, exploiting the much-reduced sagging in shorter channels.

\subsection{Summary}

Transverse ITP is still in the infant stages of development, although this document should serve as a sufficient proof-of-concept. If the sagging electric field and the wandering gel-boundary issues can be resolved, the path to verifying the models of Chapter 3 is straightforward, from which work with actual biological samples and an optimized chip design will naturally follow. 


\section{APPENDIX}

Derivation of the Reynolds Transport Theorem used in Chapter 3.

Imagine a closed surface $\mathrm{S}$ enclosing volume $\mathrm{V}$. The total amount of a certain ion (i) within $\mathrm{V}$ is

$$
\int_{V} \rho_{i} d V
$$

The rate at which this enclosed amount changes is

$$
\frac{d}{d t} \int_{V} \rho_{i} d V
$$

And is equal to the rate at which the ion crosses the surface $S$ (assuming that the ion is not consumed in any chemical reactions within V). There are three methods by which the ion of interest can cross S: electrophoresis, advection, and diffusion. These processes are quantified as

$$
\begin{aligned}
& -\oint_{S} \rho_{i} v_{i} \vec{E} \bullet d \vec{s} \\
& -\oint_{S} \rho_{i} \vec{v} \bullet d \vec{s} \\
& \oint_{S} D_{i} \vec{\nabla} \rho_{i} \bullet d \vec{s}
\end{aligned}
$$

And thus 


$$
\frac{d}{d t} \int_{V} \rho_{i} d V=-\oint_{S} \rho_{i} v_{i} \vec{E} \bullet d \vec{s}-\oint_{S} \rho_{i} \vec{v} \bullet d \vec{s}+\oint_{S} D \bar{\nabla} \rho_{i} \bullet d \vec{s}
$$

By exploiting the magic of partial derivatives, and Gauss's Theorem, Equation A.6 can be rewritten as

$$
\int_{V} \frac{\partial}{\partial t} \rho_{i} d V=-\oint_{V} \bar{\nabla} \bullet\left(\rho_{i} v_{i} \vec{E}\right) d V-\oint_{V} \bar{\nabla} \bullet\left(\rho_{i} \vec{v}\right) d V+\oint_{V} \bar{\nabla} \bullet\left(D_{i} \bar{\nabla} \rho_{i}\right) d V
$$

Combining all of these under one integral gives

$$
\int_{V}\left\{\frac{\partial}{\partial t} \rho_{i}+\bar{\nabla} \bullet\left(\rho_{i} v_{i} \vec{E}\right)+\bar{\nabla} \bullet\left(\rho_{i} \vec{v}\right)-\bar{\nabla} \bullet\left(D_{i} \bar{\nabla} \rho_{i}\right)\right\} d V=0
$$

Since V is chosen arbitrarily, the integrand itself must be identically zero.

$$
\frac{\partial}{\partial t} \rho_{i}+\bar{\nabla} \bullet\left(\rho_{i} v_{i} \vec{E}\right)+\bar{\nabla} \bullet\left(\rho_{i} \vec{v}\right)-\bar{\nabla} \bullet\left(D_{i} \bar{\nabla} \rho_{i}\right)=0
$$

And thus we have arrived at the Ion Transport equation, which separately applies to each species in solution. 


\section{REFERENCES}

1 Raymond, Daniel E., Andreas Manz, and H. Michael Widmer. "Continuous Sample Pretreatment Using a Free-Flow Electrophoresis Device Integrated onto a Silicon Chip." Analytical Chemistry 66.18 (1994): 2858-865.

2 Kohlheyer, Dietrich, Jan C. T. Eijkel, Stefan Schlautmann, Albert Van Den Berg, and Richard B. M. Schasfoort. "Microfluidic High-Resolution Free-Flow Isoelectric Focusing." Analytical Chemistry 79.21 (2007): 8190-198.

${ }^{3}$ Kohlheyer, Dietrich, Geert A. J. Besselink, Stefan Schlautmann, and Richard B. M. Schasfoort. "Free-flow Zone Electrophoresis and Isoelectric Focusing Using a Microfabricated Glass Device with Ion Permeable Membranes." Lab on a Chip 6.3 (2006): 374.

4 Squires, Todd M. "Microfluidics: Fluid Physics at the Nanoliter Scale." Reviews of Modern Physics 77.3 (2005): 977-1026.

${ }^{5}$ Alexandre Persat, Matthew E. Suss and Juan G. Santiago. "Basic principles of electrolyte chemistry for microfluidic electrokinetics. Part II: Coupling between ion mobility, electrolysis, and acid-base equilibria." Lab on a Chip, 9 (2009): 2454-2469.

6 Schoch, Reto B., Mostafa Ronaghi, and Juan G. Santiago. "Rapid and Selective Extraction, Isolation, Preconcentration, and Quantitation of Small RNAs from Cell Lysate Using On-chip Isotachophoresis." Lab on a Chip 9.15 (2009): 2145.

7 Persat, Alexandre, Lewis A. Marshall, and Juan G. Santiago. "Purification of Nucleic Acids from Whole Blood Using Isotachophoresis." Analytical Chemistry (2009): 091015124656029.

8 Jung, Byoungsok, Rajiv Bharadwaj, and Juan G. Santiago. "On-Chip Millionfold Sample Stacking Using Transient Isotachophoresis." Analytical Chemistry 78.7 (2006): 2319-327.

9 Samuel L. Penrod, Terese M. Olson, Stanley B. Grant. "Whole Particle Microelectrophoresis for Small Viruses". Journal of Colloid and Interface Science, 173 (1995) 521-523.

10 Supreet S. Bahga, Moran Bercovici, and Juan G. Santiago. Supplementary Information :Ionic Strength Effects on Electrophoretic Focusing and Separations.

11 Hatch, Anson V., Amy E. Herr, Daniel J. Throckmorton, James S. Brennan, and Anup K. Singh. "Integrated Preconcentration SDS-PAGE of Proteins in Microchips Using Photopatterned Cross-Linked Polyacrylamide Gels." Analytical Chemistry 78. 14 (2006): 4976-984. 
12 Chenlu Hou and Amy E. Herr. "Ultrashort Separation Length Homogeneous Electrophoretic Immunoassays Using On-Chip Discontinuous Polyacrylamide Gels." Analytical Chemistry , 82 .8 (2010): 3343-3351.

${ }^{13}$ Advanced Radiation Corporation.

14 Alexandre Persat, Robert D. Chambers and Juan G. Santiago. "Basic principles of electrolyte chemistry for microfluidic electrokinetics. Part I: Acid-base equilibria and $\mathrm{pH}$ buffers." Lab on a Chip, 9 (2009): 2437-2453.

15 "Buffer Solution." Wikipedia, the Free Encyclopedia. Web. 07 Apr. 2011. $<$ http://en.wikipedia.org/wiki/Buffer_solution>. 